\title{
Development of a Nonhydrostatic Version of the Mesoscale-Convection-Resolving Model and its Application to the Eyewall and Spiral Rainbands of Tropical Cyclones
}

\author{
Masanori YAMASAKI \\ Research Institute for Global Change, Japan Agency for Marine-Earth Science and Technology, Yokohama, Japan
}

(Manuscript received 14 January 2010, in final form 30 April 2010)

\begin{abstract}
A numerical model in which the effects of cumulus convection are incorporated as the subgrid-scale and mesoscale organized convection is resolved by the grid (mesoscale-convection-resolving model, MCRM) was developed in the 1980s with an intention of improving the parameterization schemes for moist convection, which had been used since the 1960s. As in many numerical models with parameterization in the 1980s, hydrostatic equilibrium was assumed. The present paper describes a nonhydrostatic version of the MCRM, with some modifications of the subgrid-scale effect formulation used in the hydrostatic MCRM.

Numerical experiments are performed to get some evaluation of the performance of the nonhydrostatic version of the MCRM through the comparison of the results in two cases with and without the effects of the subgrid-scale cumulus convection. Although the most efficient horizontal grid size of the MCRM ranges from about $20 \mathrm{~km}$ to $5 \mathrm{~km}$, only the results of a $15-\mathrm{km}$ grid case are presented in this paper. The initial condition used in the numerical experiments is idealized (simplified). However, such numerical experiments can be considered useful to understand the behavior of spiral rainbands and eyewall convection in well-developed tropical cyclones and to evaluate the model validity. The subgrid-scale effects in the nonhydrostatic MCRM are not so important as those in the hydrostatic MCRM. However, it is shown that the eyewall and spiral rainbands are not simulated well unless the subgrid-scale effects are incorporated.
\end{abstract}

\section{Introduction}

This paper describes a mesoscale-convectionresolving, nonhydrostatic model, which can be used for a coarse horizontal grid size of about 5$20 \mathrm{~km}$. The development of this model is made by incorporating a scheme (or formulation) as to the effect of the subgrid-scale cumulus convection into a nonhydrostatic model of Yamasaki (2004)*. The most important aspect of the model development is to incorporate the scheme that has been used in the author's mesoscale-convection-resolving hydrostatic model in these many years. Hereafter, mesoscale-convection-resolving hydrostatic and

Corresponding author: Masanori Yamasaki, Research Institute for Global Change, Japan Agency for Marine-Earth Science and Technology, 3173-25 Showa-machi, Kanazawa-ku, Yokohama 236-0001, Japan.

E-mail: yamas@jamstec.go.jp

(C) 2010, Meteorological Society of Japan nonhydrostatic models are referred to as hydrostatic MCRM and nonhydrostatic MCRM, respectively.

The hydrostatic MCRM was developed in the middle of the 1980s (Yamasaki 1986, 1987) with an intention of improving the parameterization schemes of moist convection that had been used in tropical cyclone (hereafter, TC) models in the 1960s and 1970s (Kuo 1965; Ooyama 1964, 1969; Yamasaki 1968a, b, c; Anthes et al. 1971; Anthes 1972; Kurihara and Tuleya 1974; and many others). One of the important shortcomings of many TC models which the author recognized at that time is that internal gravity waves are excited to an unrealistic extent under certain conditions when the balance assumption used by Ooyama $(1964,1969)$ is removed (Syono and Matsuno 1963, unpublished;

* When the model is used for a horizontal grid size of $100 \mathrm{~m}-1 \mathrm{~km}$, it can be referred to as a cumulusconvection-resolving model. 
Syono and Yamasaki 1966; Yamasaki 1968a, b, c). In this connection, the author interpreted the spiral rainbands obtained by three-dimensional models in the 1970s (Anthes et al. 1971; Kurihara and Tuleya 1974) as the internal gravity waves excited by parameterized convective heating. It appears that the propagation of the calculated rainbands was too fast and the rainbands were not similar to the observed rainbands with respect to not only propagation speed but also vertical structure. The most serious concern in performing numerical experiments of Yamasaki (1968a, b, c) was to suppress the unrealistic gravity waves by taking account of the appropriate vertical distribution of parameterized convective heating on the basis of the linear theory of Syono and Yamasaki (1966).

Another important point in the parameterization schemes is that the development of a TC in a numerical model strongly depends on the vertical distribution of convective heating, particularly at low levels (Syono and Yamasaki 1966; Yamasaki 1969). In addition, vertical distribution was recognized to be important not only in TCs but also in tropical disturbances (such as easterly waves) and equatorial waves (Yamasaki 1969, 1971). However, what determines the vertical distribution in TCs in nature was not understood, and therefore, parameterization schemes had large uncertainty with respect to this important aspect.

These recognitions in the late 1960s and the early 1970s led the author to study TCs and other phenomena in which cumulus convection plays an important role by using a cumulus-convectionresolving model (Yamasaki 1975, 1977a, b, 1979, 1983, 1984). Some of the important results obtained from these studies (including a more general understanding of conditional instability of the second kind (CISK)) are summarized in Yamasaki (2007a).

The most important recognition from the studies with the cumulus-convection-resolving model $\left(\mathrm{CCRM}^{*}\right)$ was that it should be very important to

* CCRM corresponds to the cloud-resolving model (CRM), which was originally defined as a model with a horizontal grid size of $1 \mathrm{~km}$ or less. Subsequently, some researchers have used the term CRM also for a coarse-grid model with a several kilometer grid. The term cloud resolving can be used for a wide range of clouds. Therefore, the author has used the term cumulus-convection-resolving for a fine-grid model. Since the MCRM also intends to resolve "clouds," it can be considered that both the CCRM and the MCRM belong to the CRM defined in a wide sense. resolve "mesoscale organized convection" by the grid when one uses a coarse-grid model that cannot resolve cumulus convection, particularly, in cases when external forcing for moist convection is not strong. A typical example of convection under a strong external forcing is the eyewall convection of TCs, as manifested by frictional convergence. One of the examples under a weak forcing is convective activity in the TC formation (or genesis) process.

The term mesoscale organized convection is a general term that has been widely used at least since the 1970s. Observed convection or convective systems that have the mesoscale have been called mesoscale organized convection. The author has used this term as having a more specific meaning since Yamasaki (1983, 1984). Hereafter, the mesoscale organized convection specified below is referred to as mesoscale convection, and it is abbreviated as MC. Although MC is one of the organized forms of cumulus convection, it is important that $\mathrm{MC}$ can be considered as the basic organized form of cumulus convection. The time scale of MC takes a wide range from a few hours to about $10 \mathrm{~h}$ (or more). Although the most typical time scale is about $3 \mathrm{~h}$ under many conditions, the time scale can be longer, depending on the external forcing such as frictionally controlled flow, vertical shear of the environmental flow, and cloud microphysical processes (Yamasaki 1983, 1984). A physical interpretation of the time scale of MC (including the importance of rainwater evaporation and cold pool) was also given. The most important recognition from the viewpoint of numerical modeling is that $\mathrm{MC}$ is the basic mode of the mesoscale, and it is important to resolve MC in the case of a coarsegrid model in which cumulus convection cannot be resolved. The author has referred to a numerical model that intends to resolve MC as MCRM.

It can be considered that the observed mesoscale organized convection in the general sense is MC itself in some cases and an organized form of MC in other cases. The rainband (and cloud cluster) is an example of the organized forms of MC. It has been known since the 1950s that the spiral rainband of the TC consists of mesoscale echo cells (Tatehira 1961, 1962). It can be considered that MC corresponds to such a mesoscale echo cell in many cases. The mesoscale precipitation feature (MPF), which was described by Leary and Houze (1979), may correspond to MC. Since individual MCs interact in many cases, the observed shape and behavior of MC have large variability. In addition, since the 
time scale of $\mathrm{MC}$ takes a wide range, it appears that many of the spectral analysis and subjective inspection of observed convection have not revealed the dominance of the mesoscale with a particular time scale. These appear to be the reasons why the existence of the basic mode of the mesoscale, such as $\mathrm{MC}$, has not been clearly recognized although some observational studies might have suggested its existence.

It should also be remarked that the importance of MC had not been taken into account in any parameterization schemes of moist convection before Yamasaki $(1986,1987)$. In these schemes, the effects of cumulus convection and $\mathrm{MC}$ had not been clearly distinguished. In other words, it appears that the most important aspect in constructing a parameterization scheme, i.e., what is to be parameterized and what is to be resolved by the grid of a numerical model, was not clearly recognized. This may also be understood from the fact that recent studies have applied the parameterization schemes used in TC models and large-scale models (including numerical weather prediction models and general circulation models) to mesoscale phenomena such as rainbands and cloud clusters of the mesoscale. In the MCRM, only the effect of cumulus convection is incorporated as the subgrid-scale*, and it is intended that $\mathrm{MC}$ is resolved by the grid although the scale separation between cumulus convection and $\mathrm{MC}$ is not necessarily very clear in some cases.

The MCRM was developed in the 1980 s, as mentioned above. At that time, many TC models, numerical weather prediction models, and general circulation models were hydrostatic models. The development of a hydrostatic MCRM was a natural choice as the first step. Since cumulus convection is treated as the subgrid scale in the MCRM, the hydrostatic approximation is justified to a fair degree, particularly, for a qualitative understanding of TCs and other large-scale phenomena. Although some researchers have argued that a nonhydrostatic

* The scheme that represents the effect of subgrid-scale cumulus convection can be called a parameterization scheme in a wide sense. In case of the MCRM, the term parameterization can be used to imply that the effect of the subgrid-scale cumulus convection is incorporated in terms of the grid-resolved variables. The use of this term can be considered valid even in the MCRM in which scale separation between the subgrid-scale and the grid-resolved is not very distinct although the author has rather avoided the use of this term since the 1990s (Yamasaki 1996). model is required for a horizontal grid size of about $10 \mathrm{~km}$ or less, the author has considered that it depends on what is explicitly treated (or resolved) and what is treated as the subgrid scale (or parameterized). It is obvious that the nonhydrostatic MCRM is better than the hydrostatic MCRM in many respects. However, the author considers that the hydrostatic MCRM has contributed to better understanding of many aspects of TCs and other phenomena in these 20 years (and even in the future).

Since it is intended that MC be resolved in the MCRM, the mixing ratios of cloud water and rainwater are included as prognostic variables. This was one of the most important differences between the hydrostatic MCRM (Yamasaki 1986, 1987) and other hydrostatic models (including large-scale models and general circulation models) in the 1980s and 1990s (and even in recent years). The inclusion of cloud water and rainwater was based on the result from the CCRM that the cooling due to rainwater evaporation and a cold pool play important roles in TCs. This was one of the most important aspects of the improvement of the parameterization schemes. In connection with this, the subgrid-scale rainwater mixing ratio was also included as one of prognostic variables because the subgrid-scale rainwater can play an important role in the formation (and successive formation) of MC. On the other hand, the subgrid-scale cloud water mixing ratio was treated diagnostically in the early version of the MCRM (Yamasaki 1986, 1987), as done in the parameterization schemes since the 1970s (Ooyama 1971; Arakawa and Schubert 1974). In a revised version (Yamasaki 2001), not only the subgrid-scale rainwater but also the subgrid-scale cloud water are treated prognostically. The prognostic treatment of the subgrid-scale cloud water has contributed to much improvement of the cloud water distribution (Yamasaki 2001, 2005b).

There are other several important features in the MCRM. Some of them are summarized in Yamasaki (2007a), and some details are described in Section 2. The hydrostatic MCRM has been used to study the mesoscale structure of TCs (Yamasaki 1986, 1987, 2005b), TC formation (Yamasaki 1988, 1989a, b, 2006a, b), TC motion (Yamasaki 1992), cloud clusters associated with Baiu fronts (Yamasaki 2002, 2005a, 2007b, 2008), and the diurnal variation of rainfall over a large island in the equatorial area (Yamasaki, presented at the spring meeting of the Meteorological Society of Japan in 2007). Although the ice-phase processes have not 
been taken into account in the hydrostatic MCRM yet, this model will be still useful in coming years. In particular, it can be expected that the MCRM will be very important in the near future when the global warming effects on TCs and other phenomena will be widely studied with a horizontal grid size of $20 \mathrm{~km}$ or so.

Now that the author has used the hydrostatic MCRM to understand many aspects of TCs and other phenomena in these 20 years, it can be considered that it is time when a nonhydrostatic version of the MCRM should be developed. The main reason for such development is that a fine-mesh nonhydrostatic model (CCRM) is still expensive, and its application tends to be limited to problems that can be studied with a small domain. The nonhydrostatic MCRM should have wide application if an appropriate model is to be developed. Even in the future when a fine-mesh nonhydrostatic global model (global CCRM) will be used, it can be expected that the coarse-grid MCRM will be still very useful for efficient research and prediction, depending on their objectives.

In the development of the nonhydrostatic MCRM, the scheme as to the effect of cumulus convection as the subgrid-scale (cumulus parameterization in a wide sense) can be improved by comparing the results from the MCRM with those from the CCRM in the same dynamical, thermodynamical, and computational frameworks. Since a 1-km grid model without any parameterization scheme is never justified in case of the hydrostatic model, results from the hydrostatic MCRM have to be compared with the results from a nonhydrostatic CCRM (as well as observations). Although qualitative comparison and some justification are possible, different frameworks with respect to the dynamical, thermodynamical, and computational aspects are likely to obscure appropriate evaluation of the parameterization scheme. Although even results from any CCRM (particularly, with a grid size of $1 \mathrm{~km}$ or so) are not necessarily reliable for a benchmark of the scheme, it can be considered important as the first step. The development of the nonhydrostatic MCRM will also help us improve the MCRMs in that results from the hydrostatic and nonhydrostatic MCRMs can be compared.

As the first step of evaluating the performance of a developed nonhydrostatic MCRM, the model is applied to the eyewall and rainbands in welldeveloped TCs. As was mentioned, the importance of resolving $\mathrm{MC}$ and that of incorporating the effect of the subgrid-scale cumulus convection depend on the intensity of the external forcing for convection. The weaker the forcing, more important is the subgrid-scale effect. Since the eyewall convection is strongly controlled by frictional convergence, the importance of incorporating the effect of the subgrid-scale may be relatively small compared to other convective systems. Probably some or many researchers have ever confirmed this. The author (e.g., Yamasaki 1996) performed numerical experiments on TCs by using hydrostatic models with and without the effect of cumulus convection, with a grid size of $10 \mathrm{~km}$. The behavior of a calculated eyewall was not bad even without the effect of cumulus convection. As for (spiral) rainbands, their behavior was considerably bad in the later stage of time integration.

In this paper, some results from numerical experiments with nonhydrostatic models are presented. A vortex with a maximum tangential velocity of $10 \mathrm{~m} \mathrm{~s}^{-1}$ is given at the initial time. The vortex develops, and an eyewall and rainbands appear in a short time. The primary objective of the numerical experiments is to show to what degree the incorporation of the effect of the subgridscale cumulus convection improves the behavior of the eyewall and rainbands. However, since we do not know the true solution of the problem, the results described in this paper are inconclusive, and this study should be considered as the first step toward the development of a better nonhydrostatic MCRM. Results from an application of the present model to the TC formation process in which the effect of the subgrid-scale cumulus convection is much more important will be reported in the subsequent paper.

\section{Description of a nonhydrostatic MCRM}

\subsection{Basic equations}

As mentioned in Section 1, the scheme that represents the effect of cumulus convection (Yamasaki 2001, 2002) is incorporated into a nonhydrostatic model of Yamasaki (2004). The model is an elastic (or compressible) version, which includes a prognostic equation for the density as well as those for the potential temperature $\theta$ and mixing ratios of water vapor and other water substances. As in the author's anelastic model (Yamasaki 1977a, b) as well as in many elastic models, potential temperature is taken to be a prognostic variable although entropy has been used by Ooyama (1990, 2001) and total energy by Satoh (2003). 
The Cartesian coordinates $(x, y, z)$ are used, where $x$ and $y$ are horizontal coordinates, and $z$ is the height used as the vertical coordinate. The equations of motion and the continuity equation for the total mass* used in the MCRM are the same as those described in Yamasaki (2004)**. The effect of cumulus convection on momentum transport is not included because a large portion of momentum transports can be described by $\mathrm{MC}$ and TC-scale circulation. The equations for $\theta$ and mixing ratios of water substances may be written as follows:

$$
\begin{aligned}
& \frac{1}{\rho_{d}} \frac{D\left(\rho_{d} \theta\right)}{D t}=\frac{1}{C_{p d}^{*} \pi}\left[Z_{\theta}-Q_{R A D}\right]+P_{\theta}+F(\theta), \\
& \frac{1}{\rho_{d}} \frac{D\left(\rho_{d} q_{V}\right)}{D t}=Z_{V}+P_{V}+F\left(q_{V}\right), \\
& \frac{1}{\rho_{d}} \frac{D\left(\rho_{d} q_{C}\right)}{D t}=Z_{C}+P_{C}+F\left(q_{C}\right), \\
& \frac{1}{\rho_{d}} \frac{D\left(\rho_{d} q_{R}\right)}{D t}=\frac{1}{\rho_{d}} \frac{\partial\left(\rho_{d} V_{R} q_{R}\right)}{\partial z}+Z_{R}+P_{R}, \\
& \frac{1}{\rho_{d}} \frac{D\left(\rho_{d} q_{I}\right)}{D t}=Z_{I}+P_{I}+F\left(q_{I}\right), \\
& \frac{1}{\rho_{d}} \frac{D\left(\rho_{d} q_{S}\right)}{D t}=\frac{1}{\rho_{d}} \frac{\partial\left(\rho_{d} V_{S} q_{S}\right)}{\partial z}+Z_{S}+P_{S}, \\
& \frac{1}{\rho_{d}} \frac{D\left(\rho_{d} q_{G}\right)}{D t}=\frac{1}{\rho_{d}} \frac{\partial\left(\rho_{d} V_{G} q_{G}\right)}{\partial z}+Z_{G}+P_{G},
\end{aligned}
$$

where

$$
\begin{aligned}
\frac{D\left(\rho_{d} A\right)}{D t}= & \frac{\partial\left(\rho_{d} A\right)}{\partial t}+\frac{\partial\left(\rho_{d} u A\right)}{\partial x}+\frac{\partial\left(\rho_{d} v A\right)}{\partial y} \\
& +\frac{\partial\left(\rho_{d} w A\right)}{\partial z}
\end{aligned}
$$

and $A$ stands for $\theta, q_{V}, q_{C}, q_{R}, q_{I}, q_{S}$, and $q_{G}$. The latter six variables indicate the mixing ratios of water vapor, cloud water, rainwater, cloud ice, snow (snow aggregate), and graupel, respectively. These predicted variables indicate the grid-resolved field. The effects of the cloud microphysical processes are denoted by $Z$ in (1)-(7), which are in-

* The total mass includes those of dry air, water vapor, cloud water, rainwater, and water substances at the solid phase, which are classified as cloud ice, snow, and graupel in Yamasaki (2004).

** The total density $\rho$ is missing in the Coriolis term in (1) of Yamasaki (2004) although the numerical model included it. cluded by a bulk method. Assumptions for $Z$ are described in Yamasaki (2004). The terms $P_{\theta}, P_{V}, P_{C}, \ldots, P_{G}$ indicate the subgrid-scale effects of cumulus convection. Notations $u$ and $v$ are the horizontal wind speeds in the $\mathrm{x}$ - and $\mathrm{y}$-directions. $w$ is the vertical velocity; $\rho_{d}$, the density of dry air; and $\pi$, Exner function. The first terms on the right hand side of (4), (6), and (7) indicate the effects of the fall of rainwater, snow, and graupel relative to the air. In these terms, $V_{R}, V_{S}$, and $V_{G}$ are the terminal velocities (positive: downward) of rainwater, snow, and graupel, respectively. The quantity $Q_{R A D}$ indicates radiative cooling effect. In this study, it is assumed to consist of a constant cooling rate of $1.0 \mathrm{~K} \mathrm{day}^{-1}$ and Newtonian cooling with a time constant of 10 days.

The last terms $F$ in (1), (2), (3), and (5) indicate the effects of subgrid-scale eddy diffusion, which is assumed to be of the following form:

$$
\begin{aligned}
& F(A)=K_{H}\left(\frac{\partial^{2} A}{\partial x^{2}}+\frac{\partial^{2} A}{\partial y^{2}}\right)+\frac{1}{\rho_{d}} \frac{\partial}{\partial z} F_{L}(A) \\
& F_{L}(A)=\left\{\begin{array}{l}
K_{V} \rho_{d} \frac{\partial A}{\partial z} \quad\left(z>z_{s}\right) \\
\rho_{d} C_{E} V\left(A_{\text {sea }}-A_{s}\right) \quad\left(z=z_{s}\right)
\end{array}\right.
\end{aligned}
$$

where $A$ stands for $\theta, q_{V}, q_{C}$, and $q_{I}$. The coefficients of the horizontal and vertical eddy diffusion are denoted by $K_{H}$ and $K_{V}$, respectively. These coefficients depend on thermal stratification and wind field, as assumed in most numerical models. However, the present author has not used such assumptions in his studies on convection and tropical cyclones so far, mainly because the use of this simple assumption has been sufficient for his discussions on these problems. The values of $K_{H}$ and $K_{V}$ are assumed to be $100 \mathrm{~m}^{2} \mathrm{~s}^{-1}$ below a height of $1 \mathrm{~km}$ and 0 above $3 \mathrm{~km}^{* * *}$ and are assumed to linearly decrease with height between $1 \mathrm{~km}$ and $3 \mathrm{~km}$. The impact of the use of a sophisticated formulation of these quantities remains to be studied. The eddy fluxes at the earth's surface (anemometer level $z_{S}$ ) are only applied to $\theta$ and $q_{V}$ (sensible and latent heat fluxes) and are included by the bulk method, as used in many models. It is well known that sensible and latent heat fluxes as well as surface friction play very important roles in TCs. In the above

*** In order to suppress gravity waves near the upper boundary $(z=24 \mathrm{~km})$ of the numerical model, a large numerical damping is included in the same form as $F(A)$ above $18 \mathrm{~km}\left(1000 \mathrm{~m}^{2} \mathrm{~s}^{-1}\right.$ or less in terms of $K_{H}$ and $K_{V}$ ). 
formulation, $V$ is the wind speed near the surface, $A_{\text {sea }}$ stands for potential temperature corresponding to the sea surface temperature or the corresponding saturation mixing ratio of water vapor, and $A_{s}$ is the value of $\theta$ or $q_{V}$ at $z_{s}$. The coefficient $C_{E}$ is taken to be 0.0015 . (The drag coefficient $C_{D}$ for momentum flux is also taken to be 0.0015 .)

A quantity concerning specific heat, $C_{p d}^{*}$ is used as an approximation in (1). It is given by

$$
C_{p d}^{*}=\left(1+\frac{C_{p V}}{C_{p d}} q_{V}\right) C_{p d}=\left(1+4.2 q_{V}\right) C_{p d},
$$

where $C_{p d}$ is the specific heat of dry air at constant pressure $\left(1004 \mathrm{~J} \mathrm{~kg}^{-1} \mathrm{~K}^{-1}\right)$, and $C_{p V}$ is the specific heat of water vapor at constant pressure $\left(4.2 \times 10^{3} \mathrm{~J} \mathrm{~kg}^{-1} \mathrm{~K}^{-1}\right)$.

In the compressible system of equations used in this study, temperature $T$ is calculated from potential temperature $\theta, \rho_{a}$, and $q_{V}$ :

$$
T=\theta^{\gamma}\left[\rho_{a} R_{d}\left(1+0.608 q_{V}\right) / p_{0}\right]^{\gamma-1},
$$

where $p_{0}$ is a reference pressure $(1,000 \mathrm{hPa}), R_{d}$ is the gas constant for dry air $\left(287.04 \mathrm{~J} \mathrm{~kg}^{-1} \mathrm{~K}^{-1}\right)$, and $\gamma$ is 1.4 , i.e., the ratio of the specific heat of dry air at constant pressure $C_{p d}$ to that at constant volume $C_{v d}\left(717 \mathrm{~J} \mathrm{~kg}^{-1} \mathrm{~K}^{-1}\right)$. The value of $\rho_{a}$, which is the sum of dry air density and water vapor density, is determined from the total density $\rho$, using the following relation:

$$
\rho_{a}=\left(1+q_{V}\right) \rho /\left(1+q_{V}+q_{l s}\right),
$$

where $q_{l s}$ is the sum of mixing ratios of water substances in liquid and solid phases.

The pressure $p$ is calculated from the equation of state:

$$
p=\rho_{a} R_{d}\left(1+0.608 q_{V}\right) T .
$$

When the unit of $\rho$ is taken to be $\mathrm{kg} \mathrm{m}^{-3}$, the unit of $p$ is $\mathrm{kg} \mathrm{m}^{-1} \mathrm{~s}^{-2}$ or $0.01 \mathrm{hPa}$. A set of equations used in the MCRM as well as Yamasaki (2004) determines $T$ and $p$ when $u, v, w, \rho, \theta, q_{V}, q_{C}, q_{R}, q_{I}$, $q_{S}$, and $q_{G}$ are determined by prognostic equations (except for a problem arising from the fact that saturation mixing ratio is a function of $T$ and $p$ ).

The left hand side of (1)-(7) $\frac{1}{\rho_{d}} \frac{D\left(\rho_{d} A\right)}{D t}$ is equal to $\frac{d A}{d t}$ because

$$
\frac{\partial \rho_{d}}{\partial t}+\frac{\partial\left(\rho_{d} u\right)}{\partial x}+\frac{\partial\left(\rho_{d} v\right)}{\partial y}+\frac{\partial\left(\rho_{d} w\right)}{\partial z}=0 .
$$

The expression in (1)-(7) indicates that the flux form is used for the advection terms, which means that water vapor, cloud water, and other water substances are conserved over a closed domain through the advection terms.

\subsection{Effects of the subgrid-scale cumulus convection}

In (1)-(7), the effects of cumulus convection treated as the subgrid-scale are denoted by $P_{\theta}$, $P_{V}, P_{C}, P_{R}, P_{I}, P_{S}$, and $P_{G}$. Following the usual way of treating the subgrid-scale effects (Yamasaki 2001), some of these quantities may be written as follows:

$$
\begin{aligned}
& P_{\theta}=\frac{L}{c_{p} \pi} C^{C}-N^{C}(\theta)+D^{C}(\theta), \\
& P_{V}=-C^{C}-N^{C}\left(q_{V}\right)+D^{C}\left(q_{V}\right), \\
& P_{C}=C^{C}-N^{C}\left(q_{C}\right)+D^{C}\left(q_{C}\right)-\left(C_{\text {onv }}\right)_{C R}^{C}, \\
& P_{R}=-N^{C}\left(q_{R}\right)+\left(C_{\text {onv }}\right)_{C R}^{C}+\left(F_{\text {all }}\right)_{R}^{C},
\end{aligned}
$$

where

$$
\begin{aligned}
& N^{C}(A)=\frac{1}{1-\sigma} \frac{1}{\rho_{d}} \frac{\partial}{\partial z}\left[\left(M^{C}-\rho_{d} \sigma w\right)\left(A^{C}-A\right)\right] \\
& M^{C}=\rho_{d} \sigma w^{C} .
\end{aligned}
$$

As for $P_{I}, P_{S}$, and $P_{G}$, the assumptions will be described in subsection 2.5. The most important quantity in (10a)-(10d) is the rate of condensation in the ascending area of the cumulus-scale, which is denoted by $C^{C}$. The notation $L$ is the latent heat of condensation. The terms $N^{C}$ indicate the effects of the vertical transport by the cumulus-scale, which is formulated with the use of $\sigma$, the area ratio of upward motion of the cumulus-scale*, and $M^{C}$, the mass flux associated with upward motion of the cumulus-scale, as well as $w$. The mass flux $M^{C}$ is defined by $(11 \mathrm{~b})$, where $w^{C}$ is the vertical velocity in the cumulus-scale ascending area. The mass flux $M^{C}$ can be referred to as "cumulus-scale mass flux." (In contrast, $\rho w$ can be called "mesoscale mass flux.") The value of $\left(A^{C}-A\right)$ in (11a) is assumed to be zero around (and below) the cloud base level. Other notations are $D^{C}$, the effect of the cumulus-scale downdraft; $\left(C_{\text {onv }}\right)_{C R}^{C}$, the cumulusscale effect of the conversion from cloud water to rainwater; and $\left(F_{\text {all }}\right)_{R}^{C}$, the cumulus-scale effect of the fall of rainwater relative to the air.

* When $\sigma$ is assumed to be sufficiently small $(\sigma \ll 1)$, the formulation $N^{C}$ is reduced to be a traditional form, which has been used since Ooyama (1971). As mentioned in Yamasaki (2001), $\sigma$ is assumed to be finite in the revised version of the MCRM. 
As is well known, the following relation is used in deriving (11a).

$$
A=\sigma A^{C}+(1-\sigma) A^{E},
$$

where $A$ is the grid-resolved quantity (overbar: omitted in this paper), $A^{C}$ is the quantity in the ascending area of the cumulus-scale, and $A^{E}$ is in the descending area*. The quantities $\theta^{C}, q_{V}^{C}, q_{C}^{C}$, and $q_{R}^{C}$, which appear in (11a), indicate the values in the cumulus-scale ascending area.

\subsection{Properties of the cumulus-scale ascending area}

The next problem is how to determine the thermodynamic properties in the cumulus-scale ascending area, $\theta^{C}$ and $q_{V}^{C}$. As for $q_{V}^{C}$, it is assumed to be equal to the saturation mixing ratio of water vapor. That is,

$$
q_{V}^{C}=q_{s a t}\left(T^{C}, p\right),
$$

where $q_{\text {sat }}$ is the saturation mixing ratio for temperature $T^{C}$ and pressure $p$. In order to determine $\theta^{C}$ and $q_{V}^{C}$, we use the following two conditions in addition to (12):

$\theta^{C}=T^{C}\left(p_{0} / p\right)^{R_{d} / c_{p}}$,

$\frac{\partial\left(M^{C} \theta_{e}^{C}\right)}{\partial z}-\tilde{\theta}_{e} \frac{\partial M^{C}}{\partial z}+E_{D} M^{C}\left(\theta_{e}^{C}-\theta_{e}^{E}\right)=0$,

where $\theta_{e}^{C}$ and $\theta_{e}^{E}$ are the equivalent potential temperature in the cumulus-scale ascending and descending areas, respectively. The condition (14) is derived from the budget consideration for equivalent potential temperature for the cumulus-scale ascending area.

In the budget consideration, the effects of turbulent entrainment and detrainment are taken into account, and it is assumed that the magnitude of turbulent detrainment is equal to that of turbulent entrainment. The coefficient $E_{D}$ is a parameter concerning turbulent entrainment, which will be explained later. The quantity $\tilde{\theta}_{e}$ in $(14)$ is chosen to be $\theta_{e}^{C}$ or $\theta_{e}^{E}$ according to entrainment or detrainment.

* The vertical velocity in the cumulus-scale descending area, which is denoted by $w^{E}$, is related to $w$ and $w^{C}$ as follows:

$$
w=\sigma w^{C}+(1-\sigma) w^{E},
$$

where the superscripts $C$ and $E$ for $w$ indicate convective cloud area and its environment, respectively. The values of $w^{E}$ are not necessarily negative. When the grid-resolved vertical velocity $w$ is strong to some extent, $w^{E}$ may be positive. The term cumulus-scale descending motion is used as an indicator of relative motion in this paper.

$$
\tilde{\theta}_{e}= \begin{cases}\theta_{e}^{C} & \left(\text { for detrainment } \partial M^{C} / \partial z<0\right), \\ \theta_{e}^{E} & \left(\text { for entrainment } \partial M^{C} / \partial z>0\right) .\end{cases}
$$

The lower boundary condition for (14) is obtained by assuming $\theta_{e}^{C}=\theta_{e}$ at $z=z_{B}$. The height of the lower boundary $z_{B}$ is taken to be a level in the upper portion of the boundary layer (such as $0.8 \mathrm{~km})$.

In the case of saturation, the equivalent potential temperature $\theta_{e}$ is usually approximated by

$$
\theta_{e}=\theta \exp \left(\frac{L q_{V}}{c_{p} T}\right)
$$

We can also use another approximate form

$$
\theta_{e}=\theta\left(1+\frac{L q_{V}}{c_{p} T}+\frac{L^{2} q_{V}^{2}}{2 c_{p}^{2} T^{2}}+\cdots\right) .
$$

However, the accuracies of the approximations $(15 \mathrm{a})$ and $(15 \mathrm{~b})$ are not good. As in Yamasaki (2001), $\theta_{e}$ can be approximated by the following form:

$$
\theta_{e}=\theta+F^{*}\left(\frac{L q_{V}}{c_{p} \pi_{L C L}}+\frac{L^{2} q_{V B}^{2}}{2 c_{p}^{2} \pi_{B} T_{B}}\right),
$$

where the subscript $B$ in $\pi_{B}, T_{B}$, and $q_{V B}$ indicates the value for the basic state, $\pi_{L C L}$ is the value of $\pi$ at the lifting condensation level in the case of unsaturation, and $F^{*}$ is a correction quantity, which is taken to be 1.09. This correction gives more accurate conservation quantity than $\theta_{e}$ given by $(15 \mathrm{a})$ and $(15 b)$. In order to determine the saturation state with much less computer time (without any significant loss of accuracy), we use $\pi$ instead of $\pi_{L C L}$ even in the unsaturated subcloud layer. The four conditions (12), (13), (14), and (15c) determine $T^{C}, \theta^{C}, \theta_{e}^{C}$, and $q_{V}^{C}$ when $M^{C}$ and $p$ are known. As mentioned later, the determination of $M^{C}$ requires the values of $T^{C}$ and $q_{V}^{C}$. Therefore, some iterations are necessary to determine these quantities.

The next problem is how to determine $C^{C}$. Three formulations were described and compared in Yamasaki (2002). The following formulation was chosen to be the best one:

$$
\frac{L}{c_{p} \pi} C^{C}=\frac{M^{C}}{\rho} \frac{\partial \theta^{C}}{\partial z}+\left(\theta^{C}-\theta^{E}\right) E,
$$

where $\theta^{E}$ can be approximated by $\theta$, and $E$ indicates entrainment. This formulation is obtained from a balance condition based on the heat budget for the cumulus-scale ascending area. That is, 
warming due to condensation is assumed to take place so as to cancel cooling due to adiabatic motion and entrainment.

It is assumed that entrainment $E$ consists of dynamic entrainment $E_{d y n}$ and turbulent entrainment $E_{T}$ :

$$
\begin{aligned}
& E=E_{d y n}+E_{T} \\
& E_{d y n}= \begin{cases}\frac{1}{\rho_{d}} \frac{\partial M^{C}}{\partial z} & \left(\partial M^{C} / \partial z>0\right), \\
0 & \left(\partial M^{C} / \partial z \leq 0\right)\end{cases} \\
& E_{T}=E_{D} \frac{M^{C}}{\rho_{d}},
\end{aligned}
$$

where turbulent entrainment is assumed to be proportional to the cumulus-scale mass flux $M^{C}$. A parameter concerning turbulent entrainment $E_{D}$ may take values ranging from 0.5 to $3.0 \times 10^{-4} \mathrm{~m}^{-1}$. In this study, $E_{D}$ is taken to be $2.0 \times 10^{-4} \mathrm{~m}^{-1}$. The assumption on $E_{T}$ is the same as that in Yamasaki (1986). (The definition of $E_{T}$ in that paper is different from (17b) by a factor of the thickness of the layer in the numerical model.)

The budget equation for equivalent potential temperature (14) can be rewritten using $E$ as follows:

$$
\frac{\partial \theta_{e}^{C}}{\partial z}=-\frac{\rho_{d} E}{M^{C}}\left(\theta_{e}^{C}-\theta_{e}^{E}\right) .
$$

In the case of $E=0, \theta_{e}^{C}$ of rising air is conserved, as expected. The value of $\theta_{e}^{E}$ in (14) and (18) can be approximated by the grid-resolved value $\theta_{e}$.

The determination of $\sigma$ in (11a) and (11b) is one of the most unknown problems. In the case of large-scale and planetary-scale phenomena, it is reasonable to assume that $\sigma$ is sufficiently small compared to unity, as has been done in most of the parameterization schemes. However, in the case of mesoscale phenomena, $\sigma$ is not sufficiently small. Nevertheless, this assumption has been used in parameterization schemes for mesoscale phenomena. In the first version of the MCRM (Yamasaki 1986), this assumption was used because the determination of $\sigma$ was a difficult problem and because this assumption would not invalidate the parameterization scheme essentially. It should be noted that the assumption (16) concerning $C^{C}$ does not depend on the assumption of $\sigma$ directly. (The mass flux $M^{C}$ also does not depend on $\sigma$ directly, as described later.) The assumption of $\sigma$ affects the model behavior through $N^{C}$, which has smaller contributions compared with $C^{C}$ in (10a)(10c). With this recognition, the use of reasonable non-zero values of $\sigma$ should not be very important but desirable in case of mesoscale phenomena, as incorporated by Yamasaki (2001). In this study, $\sigma$ is tentatively assumed to be a constant value, which does not depend on the thermodynamic field. The reasonable value of $\sigma$ may range from 0.1 to 0.3 . In this study, $\sigma$ is assumed to be 0.2 . Any improvement remains to be made.

\subsection{Assumption on the cumulus-scale mass flux}

The cumulus-scale mass flux $M^{c}$ is assumed to be essentially the same as that in Yamasaki (1986).

$$
\frac{M^{C}}{\rho_{d}}= \begin{cases}Y\left(w^{*}-w_{\min }^{*}\right) & \left(w^{*}>w_{\min }^{*}\right) . \\ 0 & \left(w^{*} \leq w_{\min }^{*}\right)\end{cases}
$$

The cloud mass flux is assumed to strongly depend on $w^{*}$, the vertical velocity at a certain level $z^{*}$ (a height around the top of the boundary layer such as $1 \mathrm{~km}$ ), but a threshold value $w_{\min }^{*}$ is introduced since Yamasaki (1988). It is a positive parameter such as $0.1 \mathrm{~m} \mathrm{~s}^{-1}$. In the case of larger values of $w_{\min }^{*}$ such as $0.2 \mathrm{~m} \mathrm{~s}^{-1}$, the eyewall is weaker, and convection outside the eyewall is also more inactive.

A proportional coefficient $Y$ in (21) is an essential quantity that determines the properties of MC. When $Y$ is written in the following form

$$
Y=Y^{*} \eta,
$$

$\eta$ is the normalized cumulus-scale mass flux, which is taken to be unity at $z^{*}$, and $Y^{*}$ is the value of $Y$ at $z^{*}$. The physical meaning of $Y^{*}$ is the ratio of the cumulus-scale mass flux to the mesoscale (gridresolved) mass flux at $z^{*}$ when $w_{\min }^{*}=0$. As for $\eta$, it is assumed to depend on $B$ as

$$
\eta=1+C_{\eta} B
$$

where $B$ is a measure of buoyancy of the rising air parcel, which is given by

$$
\begin{aligned}
B= & T^{C}\left(1+0.608 q_{V}^{C}-q_{C}^{C}-q_{R}^{C}\right) \\
& -T\left(1+0.608 q_{V}-q_{C}-q_{R}\right) .
\end{aligned}
$$

The value of a parameter $C_{\eta}$ in (23) is assumed to be 0.2 for positive values of $B$ and 1.0 for negative values. The latter allows an overshooting of convection into a layer where $-1<B<0$. The value of $C_{\eta}$ is 0 at $z^{*}$ according to the definition of $\eta$. 
The value of $Y^{*}$ strongly controls the growth of MC. (The values of $\eta$ just above the level $z^{*}$ are also very important.) The growth of MC should be sensitive to the distribution of $B$ in a layer just above the level $z^{*}$. Therefore, $Y^{*}$ is assumed to depend on $\eta^{* *}$ in the following form:

$$
Y^{*}= \begin{cases}1+C^{* *}\left(\eta^{* *}-1\right) & \left(\eta^{* *}>1\right), \\ \eta^{* *} & \left(1 \geq \eta^{* *} \geq 0\right)\end{cases}
$$

where $\eta^{* *}$ is the (averaged) value of $\eta$ in a layer of $2-3 \mathrm{~km}\left(2.5-3 \mathrm{~km}\right.$ in this study) heights, and $C^{* *}$ is a parameter, which is taken to be $0.5-1.5$. According to many numerical experiments performed so far, the properties of $\mathrm{MC}$ and a $\mathrm{TC}$ are not very different provided that the value of $C^{* *}$ takes this range. The value of $C^{* *}$ is taken to be 1.0 in this study. The assumptions (23)-(25) assure that the growth of $\mathrm{MC}$ tends to cease as the stratification in the lower layer becomes moist neutral. That is, it is assumed that the values of $Y^{*}$ and $\eta$ (and $Y$ ) approach 1.0 as $B$ in the lower layer becomes 0 .

A modification from Yamasaki (2001) is introduced. In place of (22), the following assumption is used:

$$
Y=C_{R H} Y^{*} \eta,
$$

where $C_{R H}$ is a modification parameter that depends on $R_{H}$, the relative humidity for water above $0^{\circ} \mathrm{C}$ and for ice below $0^{\circ} \mathrm{C}$. The value of $C_{R H}$ is taken to be 1.0 for $R_{H}>R_{H}^{L}, 0.0$ for $R_{H}<R_{H}^{S}$, and it is linearly interpolated for $R_{H}^{L}>R_{H}>R_{H}^{S}$ (for example, $R_{H}^{L}=80 \%$, and $R_{H}^{S}=70 \%$ ). An additional condition is imposed for a layer above the lowest level where $R_{H}$ is smaller than $R_{H}^{L}$; in the case when $R_{H}$ increases with increasing height, it is assumed that the value of $C_{R H}$ is constant in a layer where $R_{H}$ is larger than $R_{H}^{\text {ext }}$, which is an extreme value at the lowest level of a layer where $R_{H}$ increases with height. This additional condition means that convective activity is assumed to be weaker above a dry layer even if the rising air with entrainment has still strong buoyancy.

\subsection{Subgrid-scale cloud water and rainwater}

In the old version of the hydrostatic MCRM (Yamasaki 1986, 1987) as well as other models with parameterized convection (e.g., Ooyama 1971, Arakawa and Schubert 1974), $q_{C}^{C}$, the mixing ratio of cloud water in the convective cloud area, which is treated as the subgrid-scale, was determined in a diagnostic way by using the budgets of water vapor and cloud water. In the improved version (Yama- saki 2001, 2002), $q_{C}^{C}$ was treated by using a prognostic equation*. It was assumed that $q_{C}^{C}$ could be determined by the following equation:

$$
\begin{aligned}
\frac{\partial q_{C}^{C}}{\partial t}+ & u \frac{\partial q_{C}^{C}}{\partial x}+v \frac{\partial q_{C}^{C}}{\partial y}+w \frac{\partial q_{C}^{C}}{\partial z} \\
= & \frac{1}{\sigma}\left[C^{C}-\frac{M^{C}}{\rho} \frac{\partial q_{C}^{C}}{\partial z}-\left(q_{C}^{C}-q_{C}^{E}\right) E\right] \\
& -\left(A_{\text {uto }}^{C}+C_{o l l}^{C}\right)-e_{C}^{C} / \sigma-\delta q_{C}^{C} .
\end{aligned}
$$

This equation expresses the time change of $q_{C}^{C}$ due to the horizontal and vertical advections, the production of cloud water owing to condensation, the vertical advection of cloud water in the cumulusscale ascending area, entrainment, the effects of autoconversion and collection, and evaporation (denoted by $e_{C}^{C}$ ). The vertical advection term on the left hand side is not included for $M^{C}>0$. The last term $\delta q_{C}^{C}$ will be explained later. Although the horizontal advection terms cannot be accurately represented in a numerical model, it is necessary to include these terms.

On the other hand, an equation for the subgridscale rainwater $q_{R}^{C}$ may be written as follows:

$$
\begin{aligned}
\frac{\partial q_{R}^{C}}{\partial t}+ & u \frac{\partial q_{R}^{C}}{\partial x}+v \frac{\partial q_{R}^{C}}{\partial y}+w \frac{\partial q_{R}^{C}}{\partial z} \\
= & -\left(\frac{M^{C}}{\rho \sigma}-V_{R}^{C}\right) \frac{\partial q_{R}^{C}}{\partial z}-\left(q_{R}^{C}-q_{R}^{E}\right) E / \sigma \\
& +\left(A_{\text {uto }}^{C}+C_{o l l}^{C}\right)-e_{R}^{C} / \sigma-\delta q_{R}^{C} .
\end{aligned}
$$

The terms on the left hand side in (27) and (28) are not expressed by the flux form but by the advective form. The terms associated with the cloud mass flux and the terminal velocity of rainwater are also expressed by the advective form. In the numerical model, the conservation of $q_{C}^{C}$ and $q_{R}^{C}$ is not intended, but only qualitative effects are considered

* A prognostic treatment of the subgrid-scale cloud water was also made in another version of the MCRMs (Nasuno and Yamasaki 2001) in which Kuo (1965)'s scheme was modified with an intention that MC might be resolved by the grid. The model is a nonhydrostatic model, but a two-dimensional model. No further studies (such as the development of a three-dimensional version) have not been made partly because the cloud mass flux scheme is better than Kuo's scheme. An important result from that study was to show that even a parameterization with Kuo's scheme can better simulate the mesoscale structure of TCs when it is intended that MC is resolved. 
as important. An important point in this respect is the conservation of $q_{C}$ and $q_{R}$, which are expressed in the flux form in (3) and (4).

The last terms $\delta q_{C}^{C}$ and $\delta q_{R}^{C}$ in (27) and (28) indicate the effects of decreasing $q_{C}^{C}$ and $q_{R}^{C}$ at the stage after $M^{c}$ becomes 0 . Although these do not contribute to the changes in $q_{C}$ and $q_{R}$ directly, the decrease in $q_{C}^{C}$ or $q_{R}^{C}$ affects the amount of evaporation that can occur in unsaturated areas. The most significant effect of $\delta q_{C}^{C}$ is to reduce $q_{C}^{C}$, which is advected from the cumulus-scale ascending area, particularly, in the upper troposphere. It is assumed that, in a layer where $M^{C}$ is 0 , this reduction occurs at a finite rate:

$$
\delta q_{C}^{C}=\beta q_{C}^{C},
$$

where $\beta$ is taken to be $0.2-1.0 \times 10^{-3} \mathrm{~s}^{-1}$ (typically $0.5 \times 10^{-3} \mathrm{~s}^{-1}$, about $30 \mathrm{~min}$ of relaxation time). In the presence of the cumulus-scale mass flux, $\beta$ is taken to be 0 . As for $\delta q_{R}^{C}$, it is assumed to take the same form as (29a);

$$
\delta q_{R}^{C}=\beta q_{R}^{C} .
$$

In the subcloud layer (boundary layer), $\beta$ is taken to be 0 . In this layer, $q_{C}^{C}$ and $q_{R}^{C}$ decrease by evaporation; $e_{C}^{C}$ in (27), and $e_{R}^{C}$ in (28).

As for the subgrid-scale cloud ice $q_{I}^{C}$, snow (snow aggregate) $q_{S}^{C}$, and graupel $q_{G}^{C}$, it is assumed that these are zero as the first step of the model development. That is, $P_{I}, P_{S}$ and $P_{G}$ in (5)-(7) are assumed to be zero.

$$
\begin{aligned}
& q_{I}^{C}=q_{S}^{C}=q_{G}^{C}=0 \\
& P_{I}=P_{S}=P_{G}=0 .
\end{aligned}
$$

Only the warm rain processes are taken into account in the cumulus-scale ascending area. When the ice phase is included, the model becomes much more complex. The advantage of the ice-phase consideration is probably not significant in view of the uncertainties involved in other important aspects of the subgrid-scale formulations. Since cloud water and rainwater which are produced in the cumulusscale ascending area are also taken into account as the grid-resolved cloud water and rainwater, the latter can be converted to grid-resolved cloud ice, snow, and graupel through the terms $Z_{C}, Z_{R}, Z_{I}$, $Z_{S}$, and $Z_{G}$ in (3)-(7). Since the following relations hold

$$
\begin{aligned}
& q_{C}=\sigma q_{C}^{C}+(1-\sigma) q_{C}^{E} \\
& q_{R}=\sigma q_{R}^{C}+(1-\sigma) q_{R}^{E},
\end{aligned}
$$

the reduction of $q_{C}$ and $q_{R}$ due to the conversion to the ice phase means the reduction of $q_{C}^{C}$ and $q_{R}^{C}$ (or $\sigma)$ in many situations because $q_{C}^{E}$ and $q_{R}^{E}$ should not be negative. The inclusion of $P_{I}, P_{S}$, and $P_{G}$ remains to be made in the future when more important aspects of the subgrid-scale formulation will have been improved.

\subsection{Other physical processes \\ a. Conversion from cloud water to rainwater}

The rates of autoconversion and collection are given as the sum of these quantities in the cumulusscale ascending and descending areas. In the case when Kessler (1969)'s parameterization is used for autoconversion, it can be written as

$$
\begin{gathered}
A_{\text {uto }}=\sigma A_{\text {uto }}^{C}+(1-\sigma) A_{\text {uto }}^{E}, \\
A_{\text {uto }}^{C}= \begin{cases}\alpha\left(q_{C}^{C}-q_{C 0}\right) & \left(q_{C}^{C}>q_{C 0}\right) \\
0 & \left(q_{C}^{C} \leq q_{C 0}\right)\end{cases} \\
A_{\text {uto }}^{E}= \begin{cases}\alpha\left(q_{C}^{E}-q_{C 0}\right) & \left(q_{C}^{E}>q_{C 0}\right), \\
0 & \left(q_{C}^{E} \leq q_{C 0}\right)\end{cases}
\end{gathered}
$$

where $\alpha$ and $q_{C 0}$ are the autoconversion rate and the threshold mixing ratio of cloud water for autoconversion, respectively. In this study, Berry (1968)'s parameterization is used instead of Kessler's. In this case, autoconversion can occur even for small values of $q_{C}^{C}$ and $q_{C}^{E}$. Similarly, the collection rate is assumed to be as follows:

$$
\begin{gathered}
C_{\text {oll }}=\sigma C_{\text {oll }}^{C}+(1-\sigma) C_{\text {oll }}^{E}, \\
C_{\text {oll }}^{C}=929 q_{C}^{C}\left(\rho_{d} q_{R}^{C}\right)^{0.875} \\
C_{\text {oll }}^{E}=929 q_{C}^{E}\left(\rho_{d} q_{R}^{E}\right)^{0.875} .
\end{gathered}
$$

When the sum of autoconversion and collection among many processes involved in $Z_{C}$ in (3) and $Z_{R}$ in (4) are denoted by $\left(C_{\text {onv }}\right)_{C R},\left(C_{\text {onv }}\right)_{C R}^{C}$ in $(10 \mathrm{c})$ and $(10 \mathrm{~d})$ is expressed by

$$
\left(C_{\text {onv }}\right)_{C R}^{C}=A_{\text {uto }}+C_{\text {oll }}-\left(C_{\text {onv }}\right)_{C R} .
$$

In order to predict $q_{C}$ and $q_{R}$ using (3) and (4), it is not necessary to calculate $\left(C_{\text {onv }}\right)_{C R}$ and $\left(C_{\text {onv }}\right)_{C R}^{C}$, but it is sufficient to calculate only $A_{\text {uto }}$ and $C_{\text {oll }}$.

\section{b. Fall of rainwater}

The total (grid-resolved and subgrid-scale) effect of rainwater fall $R_{f}$ on the right-hand side of (4) is given by

$$
R_{f}=\frac{1}{\rho_{d}} \frac{\partial\left(\rho_{d} V_{R} q_{R}\right)}{\partial z}+\left(F_{\text {all }}\right)_{R}^{C} .
$$


On the other hand, when an expression similar to (32) is used, this quantity is given by

$$
\begin{gathered}
R_{f}=\sigma R_{f}^{C}+(1-\sigma) R_{f}^{E}, \\
R_{f}^{C}=\frac{1}{\rho_{d}} \frac{\partial\left(\rho_{d} V_{R}^{C} q_{R}^{C}\right)}{\partial z} \\
R_{f}^{E}=\frac{1}{\rho_{d}} \frac{\partial\left(\rho_{d} V_{R}^{E} q_{R}^{E}\right)}{\partial z},
\end{gathered}
$$

where $V_{R}^{C}$ and $V_{R}^{E}$ the terminal velocities (positive: downward) of rainwater in the cumulus-scale ascending and descending areas, respectively. From (34) and (35), the second term on the right-hand side of (34) is given by

$$
\begin{aligned}
\left(F_{\text {all }}\right)_{R}^{C}=\frac{1}{\rho_{d}}[ & \sigma \frac{\partial\left(\rho_{d} V_{R}^{C} q_{R}^{C}\right)}{\partial z}+(1-\sigma) \frac{\partial\left(\rho_{d} V_{R}^{E} q_{R}^{E}\right)}{\partial z} \\
& \left.-\frac{\partial\left(\rho_{d} V_{R} q_{R}\right)}{\partial z}\right] .
\end{aligned}
$$

In another expression, it is given by

$$
\begin{aligned}
\left(F_{\text {all }}\right)_{R}^{C}= & \frac{1}{\rho_{d}} \frac{\partial}{\partial z}\left[\sigma \rho_{d}\left(V_{R}^{C}-V_{R}\right)\left(q_{R}^{C}-q_{R}\right)\right. \\
& \left.+(1-\sigma) \rho_{d}\left(V_{R}^{E}-V_{R}\right)\left(q_{R}^{E}-q_{R}\right)\right] .
\end{aligned}
$$

These expressions are exactly equivalent. However, in a numerical model, slight differences may arise. The total effect of rainwater fall in (4) can be incorporated using either (35) with (35a) or (34) with (36b).

\section{c. Downdraft}

The effects of the subgrid-scale downdraft in (10a)-(10c) are assumed to take the same form as that for the subgrid-scale vertical eddy diffusion $F$

$$
D^{C}(A)=\frac{1}{\rho_{d}} \frac{\partial}{\partial z}\left(K_{C} \rho_{d} \frac{\partial A}{\partial z}\right),
$$

where $A$ stands for $\theta, q_{V}$, and $q_{C}$. It is assumed that the coefficient $K_{C}$ depends on the vertical velocity and the subgrid-scale rainwater at $z=z^{*}$.

$$
K_{C}= \begin{cases}K_{D} \frac{w^{*}}{w_{1}}\left(\frac{q_{R}^{C^{*}}}{q_{R 1}}\right)^{1 / 2} & \left(w^{*}>0\right) \\ 0 & \left(w^{*} \leq 0\right),\end{cases}
$$

where $w_{1}=1 \mathrm{~m} \mathrm{~s}^{-1}$ and $q_{R 1}=1 \mathrm{~g} \mathrm{~kg}^{-1}$. That is, $K_{D}$ means the value of $K_{C}$ in the case when $w=1 \mathrm{~m} \mathrm{~s}^{-1}$ and $q_{R}^{C}=1 \mathrm{~g} \mathrm{~kg}^{-1}$ at $z=z^{*}$. It is probably reasonable to assume that the value of
$K_{D}$ is 0 well above $2 \mathrm{~km}$, and it depends on height below this level. It should also depend on the value of $\sigma$. Assumption (38) has been used since Yamasaki (1986). Although no attempts to improve this assumption have been made, it remains to be studied. In the numerical model used in this study, the value of $K_{D}$ is taken to be $200 \mathrm{~m}^{2} \mathrm{~s}^{-1}$ at $z=1.0 \mathrm{~km}, 500 \mathrm{~m}^{2} \mathrm{~s}^{-1}$ at $0.6 \mathrm{~km}$, and $300 \mathrm{~m}^{2} \mathrm{~s}^{-1}$ at $0.3 \mathrm{~km}$.

\section{d. Condensation and evaporation}

Since the values of $\theta, \theta^{C}$, and $\theta^{E}\left(q_{V}, q_{V}^{C}\right.$, and $\left.q_{V}^{E}\right)$ are generally different, it may be desirable to determine saturation conditions for the cumulusscale ascending and descending areas separately. However, the saturation or unsaturation is determined for the grid-resolved variables $\theta$ and $q_{V}$ (in addition to the saturation condition (12) for the cumulus-scale ascending area). When the following condition is produced by physical processes other than condensation and evaporation,

$$
q_{V}>q_{\text {sat }}(T, p),
$$

$T$ and $q_{V}$ (and condensation amount) are determined so that it may be just saturated. The effect of condensation is included as part of $Z_{V}$ in (2) and $Z_{C}$ in (3).

When it is unsaturated, evaporation may occur. In this situation, cloud water in the cumulus-scale cloud area is not evaporated. Only $\left(q_{C}-\sigma q_{C}^{C}\right)$ can evaporate (under the condition that it does not become supersaturated). In the subcloud layer (boundary layer), $q_{C}^{C}$ can evaporate, and $e_{C}^{C}$ in (27) is positive. Similarly, evaporation of rainwater is allowed for $Q_{R}=\left(q_{R}-\sigma q_{R}^{C}\right)$ in areas where the cumulus-scale clouds exist. As in Yamasaki (1986), the evaporation rate is assumed to be

$$
e_{R}=4.32\left(q_{s a t}-q_{V}\right)\left(\rho_{d} Q_{R}\right)^{0.65} .
$$

This effect is included as part of $Z_{V}$ in (2) and $Z_{R}$ in (4). In the subcloud layer, $Q_{R}$ in (39) is taken to be $q_{R}$. That is, it is assumed that $q_{R}^{C}$ can evaporate in this layer. In this situation, $e_{R}^{C}$ in (28) is positive.

As for the ice-phase processes, only the gridresolved variables are treated, as mentioned in subsection 2.5. The details of the ice-phase processes (including condensation and sublimation of cloud ice $Z_{I}$ in (5)) are described in Yamasaki (2004). In this study, a modification is made for the terminal velocities of snow (snow aggregate) and graupel. Although no definite reason can be given, the author feels that the terminal velocity of snow is too 
large and that of graupel is too small in Yamasaki (2004). Therefore, factors 0.8 and 1.2 are multiplied for the terminal velocities of snow and graupel, respectively. Another modification is that the threshold value for autoconversion from cloud ice to snow is assumed to be $0.3 \mathrm{~g} \mathrm{~kg}^{-1}$ instead of $0.5 \mathrm{~g} \mathrm{~kg}^{-1}$ because of the large grid size used. These modifications alter vertical distributions of snow and graupel. However, the results described in this paper are not qualitatively modified.

\section{e. Additional remarks}

As mentioned in subsection 2.1 (and well known), the heat flux at the sea surface is very important for the development and structure of TCs. In this study, the amount of heat (sensible and latent heat) supplied from the sea surface is assumed to be instantaneously distributed in the lowest kilometer of the model $(0<z<1 \mathrm{~km})$. In the numerical model used in this study, the supplied heat is partitioned with a ratio of $0.50,0.35$, and 0.15 for three layers, $(0<z<0.3 \mathrm{~km}), \quad(0.3 \mathrm{~km}<z<0.6 \mathrm{~km})$, and $(0.6 \mathrm{~km}<z<1.0 \mathrm{~km})$, respectively. This ratio as well as the vertical eddy diffusion coefficient $K_{V}$ affects the behavior of computed TCs. In this respect, it should be remarked that the most appropriate formulation of the effects of cumulus convection depends on this ratio as well as the vertical eddy diffusion formulation (parameterization). The former also depends on the formulation of the cloud microphysical processes. Although it is natural that improvement of a TC model requires improvements of these three formulations, what should be emphasized is that these three improvements are more or less interrelated.

\section{Numerical experiments}

\subsection{Experimental design}

In this study, results of numerical experiments with and without the effects of the subgrid-scale cumulus convection are compared to get some evaluation of the MCRM performance. Only the comparison made for a horizontal grid size of $15 \mathrm{~km}$ is presented in this paper. In addition, comparison is made primarily for the eyewall and spiral rainbands in well-developed TCs. For this purpose, an axisymmetric vortex with a maximum tangential velocity of $10 \mathrm{~m} \mathrm{~s}^{-1}$ is imposed at the initial time on the resting atmosphere in which neither environmental flow such as the trade easterlies nor other disturbances such as the subtropical high are present. The radial profile of the tangential velocity is given by

$$
\begin{gathered}
v_{\lambda}=\left\{\begin{array}{lr}
2 v_{\max }\left\{\frac{r}{r_{m}} /\left[1+\left(\frac{r}{r_{m}}\right)^{3}\right]-C\left(\frac{r}{r_{0}}\right)^{2}\right\} & \left(r<r_{0}\right), \\
0 & \left(r>r_{0}\right),
\end{array}\right. \\
C=\frac{r_{0}}{r_{m}} /\left[1+\left(\frac{r_{0}}{r_{m}}\right)^{3}\right],
\end{gathered}
$$

where $v_{\max }$ and $r_{m}$ are the approximate values of the maximum tangential velocity and the radius of the maximum wind (RMW), respectively, and $r_{0}$ is the radius of the area of positive tangential velocity. In the numerical experiments, these values are taken to be $10 \mathrm{~m} \mathrm{~s}^{-1}, 100 \mathrm{~km}$, and $700 \mathrm{~km}$, respectively. For these values, the maximum tangential velocity is $10.6 \mathrm{~m} \mathrm{~s}^{-1}$, and RMW is $80 \mathrm{~km}$. The radial profile is a modified form of that used by Kurihara and Tuleya (1974).

The vertical profile of the initial tangential velocity is such that $v_{\max }$ decreases linearly with increasing height above a height of $1.3 \mathrm{~km}$, and it is 0 above $12 \mathrm{~km}$. The center of the initial vortex is placed at $\left(x_{0}=150 \mathrm{~km}, y_{0}=-350 \mathrm{~km}\right)$ in the Cartesian coordinates used in this study because the vortex moves in the northwest-north directions because of beta-drift. The pressure and density fields are determined so that the gradient wind and hydrostatic equilibrium may be satisfied.

The vertical distribution of the temperature in the undisturbed state is taken to be similar to that of the mean tropical atmosphere in the northwestern Pacific in summer. For the numerical model used in this study, which has 30 layers, the temperatures at these layers are given in Table 1 .

As for the moisture field, the relative humidity is specified. Table 1 also shows the relative humidity in the initial vortex center. The relative humidity is not taken to be uniform in the horizontal direction but taken to be higher in the central area of the vortex. The relative humidity in the surrounding area, which is far from the vortex center, is taken to be $80 \%$ of the value shown in Table 1 . This area is given by $\left|x-x_{0}\right|>x_{W E}$ and $y-y_{0}>y_{N}$ and $y-y_{0}<-y_{S}$. The horizontal profile in the $\mathrm{x}-$ and $y$-directions between the surrounding area and the vortex center is given by the square of the cosine. This drier condition in the surrounding area acts to suppress the convective activity in this area. In the numerical experiments presented in this paper, the values of $x_{W E}, y_{N}$, and $y_{S}$ are taken to be $500 \mathrm{~km}$. 
Table 1. Level $z$ at which temperature and thermodynamic variables are predicted, the corresponding pressure $p$, and the undisturbed (or environmental) temperature $T$ used in the numerical experiments. The values of the relative humidity $R_{H}$ indicate those for the saturated value for cloud water or cloud ice at the center of the initial vortex. The relative humidities of the environment are taken to be $80 \%$ of these values.

\begin{tabular}{lccc|cccc}
\hline$z(\mathrm{~km})$ & $p(\mathrm{hPa})$ & $T(\mathrm{~K})$ & $R_{H}(\%)$ & $z(\mathrm{~km})$ & $p(\mathrm{hPa})$ & $T(\mathrm{~K})$ & $R_{H}(\%)$ \\
\hline 22.7 & 36 & 201.8 & 44 & 6.75 & 448 & 260.9 & 79 \\
20.2 & 54 & 201.2 & 50 & 6.25 & 478 & 264.0 & 81 \\
18.1 & 78 & 200.6 & 56 & 5.75 & 509 & 267.0 & 82 \\
16.4 & 104 & 200.7 & 61 & 5.25 & 542 & 269.9 & 83 \\
14.9 & 134 & 203.5 & 65 & 4.75 & 577 & 272.8 & 84 \\
13.6 & 166 & 209.4 & 68 & 4.25 & 614 & 275.8 & 85 \\
12.5 & 197 & 217.2 & 70 & 3.75 & 653 & 278.7 & 86 \\
11.5 & 228 & 225.2 & 70 & 3.25 & 694 & 281.4 & 87 \\
10.7 & 259 & 232.3 & 70 & 2.75 & 736 & 284.1 & 88 \\
9.95 & 289 & 238.2 & 70 & 2.25 & 781 & 286.9 & 89 \\
9.30 & 317 & 243.1 & 71 & 1.75 & 828 & 289.6 & 91 \\
8.75 & 342 & 247.2 & 72 & 1.25 & 877 & 292.3 & 92 \\
8.25 & 366 & 250.8 & 73 & 0.80 & 923 & 294.8 & 90 \\
7.75 & 392 & 254.3 & 75 & 0.45 & 960 & 297.0 & 85 \\
7.25 & 419 & 257.8 & 77 & 0.15 & 993 & 299.0 & 80 \\
\hline
\end{tabular}

The more rapid decrease in the northern area than in the southern area, which corresponds to the usually observed condition that drier air in the subtropical high exists to the north of the TC formation area, may be more realistic. Such a numerical experiment indicates that this asymmetry is not important in view of the primary objective of this study.

The sea surface temperature SST is taken to be uniform in the west-east direction. It is taken to be $302 \mathrm{~K}$ from $10 \mathrm{~N}-20 \mathrm{~N}, 301 \mathrm{~K}$ at $25 \mathrm{~N}, 299 \mathrm{~K}$ at $30 \mathrm{~N}, 296 \mathrm{~K}$ at $35 \mathrm{~N}, 301 \mathrm{~K}$ at $5 \mathrm{~N}, 300 \mathrm{~K}$ at the equator, and $298 \mathrm{~K}$ at $5 \mathrm{~S}$. The position of the initial vortex center $\left(y_{0}=-350 \mathrm{~km}\right)$ corresponds to $11.8 \mathrm{~N}$. A numerical experiment with a constant SST of $302 \mathrm{~K}$ indicates that the results described in this paper are not qualitatively modified.

One of the usual ways of initiating convective activity is to give one or some buoyancy perturbations. Since the initial vortex with a maximum wind speed of $10 \mathrm{~ms}^{-1}$ is imposed in the present experiments, no buoyancy perturbation is given (except for one case described later). Under this initial condition, convection is initiated by frictional convergence. Once convection takes place, frictional convergence does not play any significant role in convective activity until the wind speed nearly attains $15-20 \mathrm{~m} \mathrm{~s}^{-1}$. Instead, the interaction between frictional inflow and outflow of cold air associated with downdrafts plays an important role, as has been emphasized in the author's previous studies.

The model domain is taken to be a square with a length of $5,160 \mathrm{~km}$ in the $\mathrm{x}$ - and $\mathrm{y}$-directions. This domain is sufficiently large to treat an isolated TC. A cyclic condition is imposed in the x-direction, and rigid walls are imposed at the northern and southern boundaries. The horizontal grid size of $15 \mathrm{~km}$ is used in the inner square domain $(1,500 \mathrm{~km} \times 1,500 \mathrm{~km})$, and it is variable in the outer domain where active convection does not occur. The number of grid points is $201 \times 201$. The top of the model domain is taken to be $24.0 \mathrm{~km}$ where the vertical velocity is assumed to be 0 . As indicated in Table 1, a 30-layer model is used. The boundary layer $(0<z<1 \mathrm{~km})$ is divided into three layers. The vertical grid size is taken to be $500 \mathrm{~m}$ above a height of $1 \mathrm{~km}$ and below $9 \mathrm{~km}$, and it is variable above.

\subsection{Effects of subgrid-scale convection}

In this subsection, a comparison is made for TCs in cases with and without the effects of subgridscale cumulus convection. The author has considered that the predicted variable that is most important in evaluating the MCRM performance is the mixing ratio of rainwater near the surface (or surface rainfall intensity).

The rainfall pattern in the early stage of the time integration takes a ring-shape in the two cases 
(under the initial condition used in this study). Figure 1 shows the surface rainfall intensity in case 1 (without the subgrid-scale effect) and case 2 (with the effect) at 12, 24, 36, 60, 72, and $84 \mathrm{~h}$. It is seen that a ring-shaped rainband propagates outward, as was noticed in an axisymmetric CCRM (with a horizontal grid size of $500 \mathrm{~m}$ ) in Yamasaki (1977a, $\mathrm{b}, 1983)$. In the three-dimensional model, the ring is somewhat distorted by the effect of the latitudinal variation of the Coriolis parameter. The phase velocity of the propagating rainband is about $2 \mathrm{~m} \mathrm{~s}^{-1}$, which is nearly the same as that obtained in the CCRM.

After $17 \mathrm{~h}$ in case 1 and after $12 \mathrm{~h}$ in case 2 , new convection occurs in the central area of the vortex. This convection creates strong positive vorticity (vertical component) near the vortex center, and thereby, strong cyclonic rotation. This feature is also similar to that noticed in the author's CCRM studies in the 1980's. The anomaly (negative values) of the central surface pressure and the maximum wind speed are indicated by numerals in Fig. 1. The difference between these quantities in case 1 and case 2 is not large for the first $48 \mathrm{~h}$, but the central surface pressure in case 2 becomes notably lower than in case 1 afterwards. Although the behavior of the outward propagating rainband in case 1 is similar to that in case 2, the rainfall pattern in the central area of the vortex (TC in the later stage) in case 2 becomes significantly different from that in case 1 . The notable deepening of the surface pressure in case 2 is related to this feature. This suggests that the effects of the subgrid-scale convection become significant at this stage.

The vertical structures of the outward propagating rainbands (not shown) as well as the phase velocities in cases 1 and 2 are qualitatively similar. These structures are also similar to that obtained by the CCRM of Yamasaki (1983), although cumulus convection and mesoscale convection on smaller scales are not simulated in cases 1 and 2 because of the coarse grid size used. This similarity comes from the common importance of the cooling due to evaporation of rainwater and resulting downdrafts and the cold pool, which are more or less simulated in cases 1 and 2 in view of those in the CCRM case.

A more important result is concerned with the difference between the properties of the rainbands in cases 1 and 2. Convection, which constitutes rainbands, grows much more slowly in case 1 than in case 2 (and in the CCRM case). The effect of the subgrid-scale cumulus convection, which is included in case 2 , is to enhance the growth of convection. Convection tends to grow much more slowly in the coarse-grid model without the subgrid-scale effect. Another important difference is seen in the height of the cloud top. The cloud top is generally lower in case 1 than in case 2 (and in the CCRM case). Probably, researchers who have been concerned with the difference between results from two models with and without the effects of subgrid-scale convection have noticed these features.

The initial condition used in cases 1 and 2 and the resulting behavior of convection at the early stage of the time integration (particularly, the persistence of the ring-shaped rainband) are not realistic in view of the actual process of observed TCs. Nevertheless, the author has considered since the 1960s that such an idealized numerical experiment is important to understand some of important properties of observed TCs and to develop a better numerical model and evaluate its performance.

It may be somewhat surprising that the outward propagation of the ring-shaped rainband is simulated even in case 1. The propagation speed and the rainfall pattern in case 1 may be more similar to those in case 2 than expected. This is primarily because the initial vortex with the maximum wind speed of $10 \mathrm{~m} \mathrm{~s}^{-1}$ is given. Some researchers may argue that the effects of the subgrid-scale convection incorporated in case 2 are underestimated.

The surface rainfall fields at $102 \mathrm{~h}$ in case 1 and case 2 are shown in the lowest right panels of Figs. 2a, b, respectively. The western and northern portions of the outward propagating rainband are still seen even at $102 \mathrm{~h}$. The southern and southeastern portions are fairly modified. Our interest is to compare the vertical structure of the cloud pattern in case 1 with that in case 2 . The total mixing ratio of cloud ice and cloud water is shown for 5 levels in Fig. 2. The most important feature of the cloud field in case 1 is that the cloud pattern in the middle and upper troposphere is fairly different from that in the lower troposphere. Compared with case 1 , the cloud patterns at various levels in case 2 are more similar. This means that many of the convective clouds in case 2 grow into the upper troposphere although some clouds are in the growing stage, and others remain as shallow clouds, whereas more clouds in case 1 are prevented from penetrating the middle troposphere. These features of the difference between the cloud patterns in case 1 and case 2 are, qualitatively, what have been expected. 
(a) case 1 (without subgrid-scale convection) (b) case 2 (with subgrid-scale)

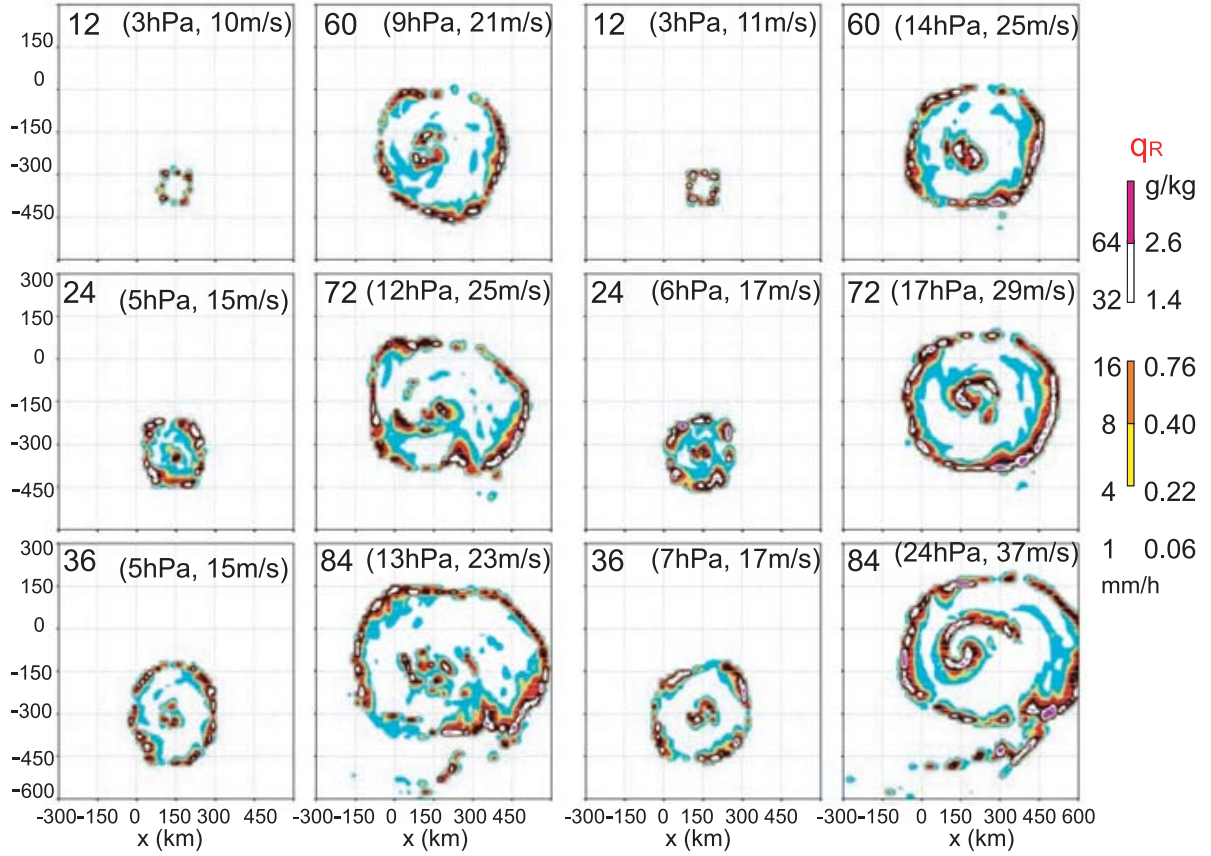

Fig. 1. Rainwater mixing ratio $q_{R}$ near the surface (or surface rainfall intensity) at $12,24,36,60,72$, and $84 \mathrm{~h}$ in case 1 and case 2 . The central surface pressure and the maximum wind speed are indicated by numerals.

(a) case 1

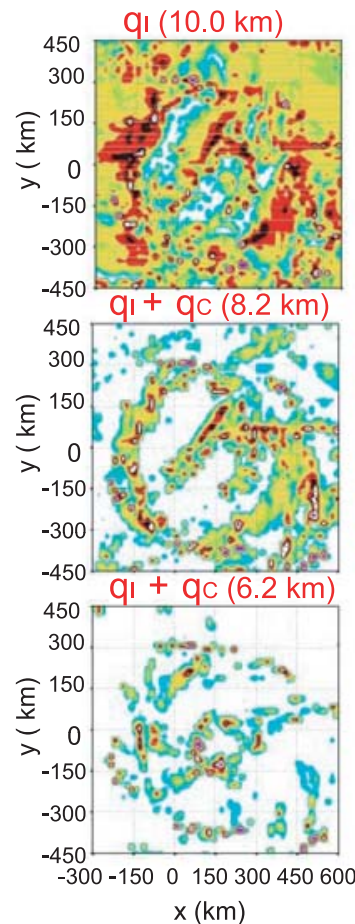

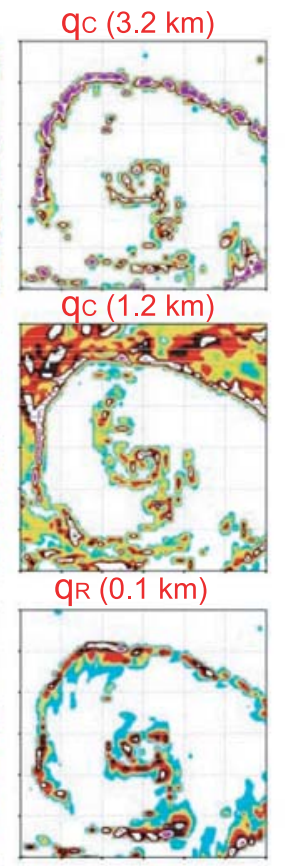
$\mathrm{x}(\mathrm{km})$ (b) case 2

$102 \mathrm{~h}$
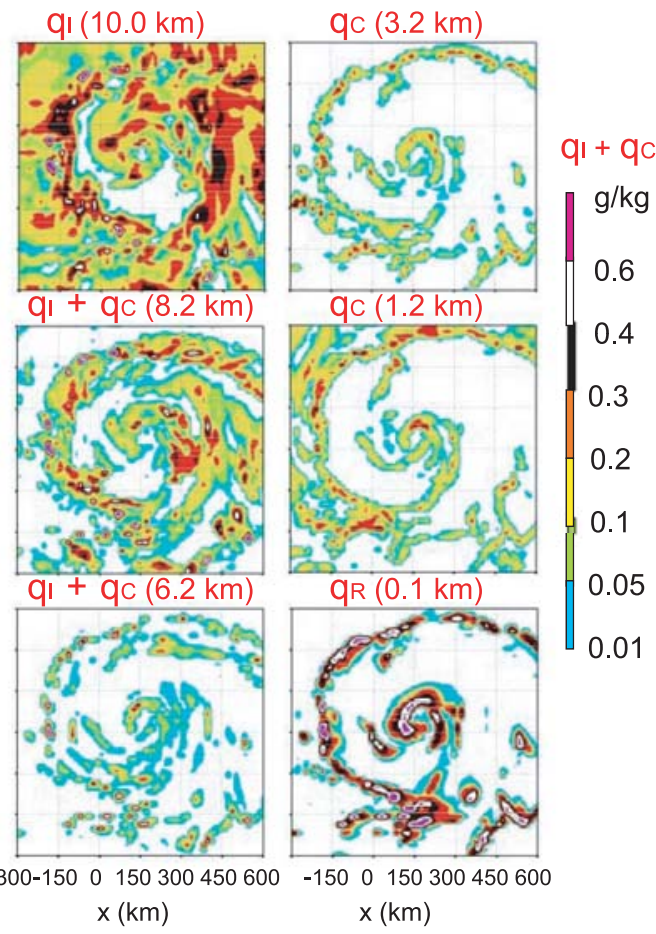

Fig. 2. Mixing ratio of the total of cloud ice and cloud water $\left(q_{I}+q_{C}\right)$ at five levels and rainwater $q_{R}$ near the surface at $102 \mathrm{~h}$ in case 1 and case 2. 
Some researchers may argue that the difference is smaller than expected and that the subgrid-scale effect in case 2 may be underestimated.

It is also seen in Fig. 2 that much more cloud water (larger mixing ratio) at low-levels $(3.2 \mathrm{~km}$ and $1.2 \mathrm{~km}$ in Fig. 2) exists in case 1 than in case 2. This is related to the above-mentioned feature that clouds do not grow easily into higher levels in case 1. As for clouds in the upper troposphere, stratiform (grid-resolved) clouds are formed around and above a height of $8 \mathrm{~km}$ or $10 \mathrm{~km}$ in both cases although the cloud top tends to be lower in case 1 , as mentioned before.

Figure 3 shows the surface rainfall intensity in case 1 and case 2 in a period from $99 \mathrm{~h}$ to $132 \mathrm{~h}$ at an interval of $3 \mathrm{~h}$. The rainbands to the west-the north-the east of the TC center continue to propagate outward (even outside the domain shown), and these decay soon or later. On the other hand, the rainbands to the south-southeast do not continue to propagate outward because a stronger southerly flow prevails and prevents the rainbands from propagating southward, or new convection is produced in this area. The most important difference between rainfall patterns in case 1 and case 2 is found in the eyewall and spiral rainbands, particularly in the later stage of the period shown in this figure. The eyewall and spiral rainbands are not simulated well in case 1 . The central surface pressure anomaly (negative value) and the maximum wind speed, which are shown by the numerals, indicate that the TC does not develop much in case 1 . On the contrary, the vortex in case 2 becomes an intense TC. The maximum anomalies of the central surface pressure in case 1 and case 2 are $-23 \mathrm{hPa}$ $(987 \mathrm{hPa})$ and $-48 \mathrm{hPa}(962 \mathrm{hPa})$, and the maximum wind speeds are $32 \mathrm{~m} \mathrm{~s}^{-1}$ and $57 \mathrm{~m} \mathrm{~s}^{-1}$, respectively. The weaker $\mathrm{TC}$ in case 1 is related to the ill-defined eyewall.

The author had not necessarily inferred that simulation of the eyewall and spiral rainbands are worse than that of the outward propagating, ringshaped rainbands in case 1 . The external dynamical forcing such as frictional convergence and frictional inflow is stronger in the eyewall and spiral rainbands than in the ring-shaped rainbands. It may be now recognized that the subgrid-scale effect of cumulus convection is not very important in determining the rainfall pattern (successive formation of convection and propagation) in case when the effect of the downdrafts and outflow of the cold air due to evaporation of rainwater plays a very important role. It appears that not only the ring-shaped rainbands but also squall-lines and band-shaped mesoscale convective systems, which are strongly controlled by the outflow of cold air associated with downdrafts, are less affected by the effects of the subgrid-scale cumulus convection than the eyewall and spiral rainbands. In this respect, it should also be remarked that the former three have strong twodimensionality, whereas the eyewall is strongly affected by asymmetric spiral rainbands. It is obvious that the grid size effect on convection is much larger in asymmetric (three-dimensional) convection than in two-dimensional convection. Therefore, the effect of subgrid-scale convection should be more important in the former case. Further studies are needed for clarification.

The surface rainfall pattern (low-level rainwater mixing ratio) after $132 \mathrm{~h}$ in case 2 is shown in Fig. 4. The pattern is shown at an interval of $1 \mathrm{~h}$. Some of the individual mesoscale cells, which constitute rainbands (and eyewall), are labeled. It is seen that most of mesoscale cells rotate cyclonically (more slowly than the cyclonic wind speed at low-levels, as in observed TCs). The lifetime of some cells exceeds $10 \mathrm{~h}$. Since the horizontal grid size is taken to be $15 \mathrm{~km}$, it is reasonable that the lifetime of these cells is generally longer than that obtained from the CCRM (3-10 h). When the grid size is taken to be smaller, it can be expected that each mesoscale cell shown in this figure is more easily replaced by a new cell that forms in its vicinity. An important point in this regard is to what extent the behavior of these cells is qualitatively realistic. The author has seen many cases of such behavior in the hydrostatic MCRM in these 20 years. The behavior in Fig. 4 is similar to that in the hydrostatic MCRM and, to a less extent, observations. Needless to say, the behavior of mesoscale cells has a large variety in the MCRM as well as in observed TCs. Fig. 4 is merely an example.

The anomaly of the central surface pressure of $-48 \mathrm{hPa}$ at $132 \mathrm{~h}$ is still increased, but very gradually. It is $-56 \mathrm{hPa}$ at $144 \mathrm{~h}$. The maximum wind speed decreases to $49 \mathrm{~m} \mathrm{~s}^{-1}$ at $137 \mathrm{~h}$, and increases to $57 \mathrm{~m} \mathrm{~s}^{-1}$ at $144 \mathrm{~h}$. This period is a nearly quasisteady or mature stage of the TC.

The behavior of mesoscale cells in case 1 (not shown) is not as realistic as that shown in Fig. 4, as suggested from Fig. 3a. An additional numerical experiment (case 3) is performed to confirm the effects of subgrid-scale convection. Figure 5 shows the surface rainfall intensity in case 3 in which the 
(a) case 1 (without the effects of subgrid-scale convection)

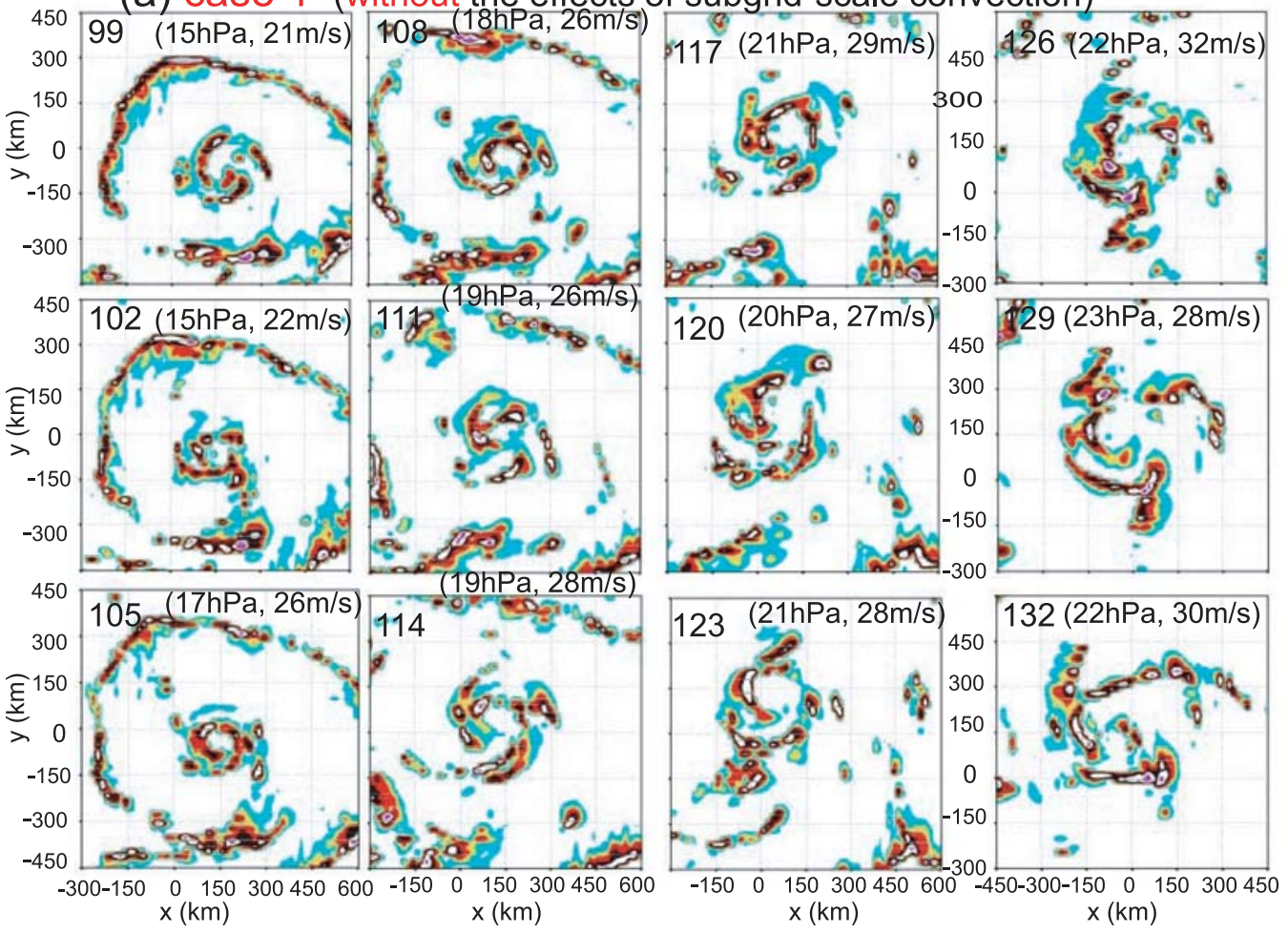

(b) case 2 (with the effects of subgrid-scale convection)



Fig. 3. Rainwater mixing ratio near the surface (or surface rainfall intensity) at an interval of $3 \mathrm{~h}$ from $99 \mathrm{~h}$ to $132 \mathrm{~h}$ in case 1 and case 2 . 



Fig. 4. Rainwater mixing ratio near the surface at an interval of $1 \mathrm{~h}$ from $133 \mathrm{~h}$ to $144 \mathrm{~h}$ in case 2 . Mesoscale cells that constitute rainbands are labeled.

case 3 (without subgrid-scale convection after $96 \mathrm{~h}$ in case 2)
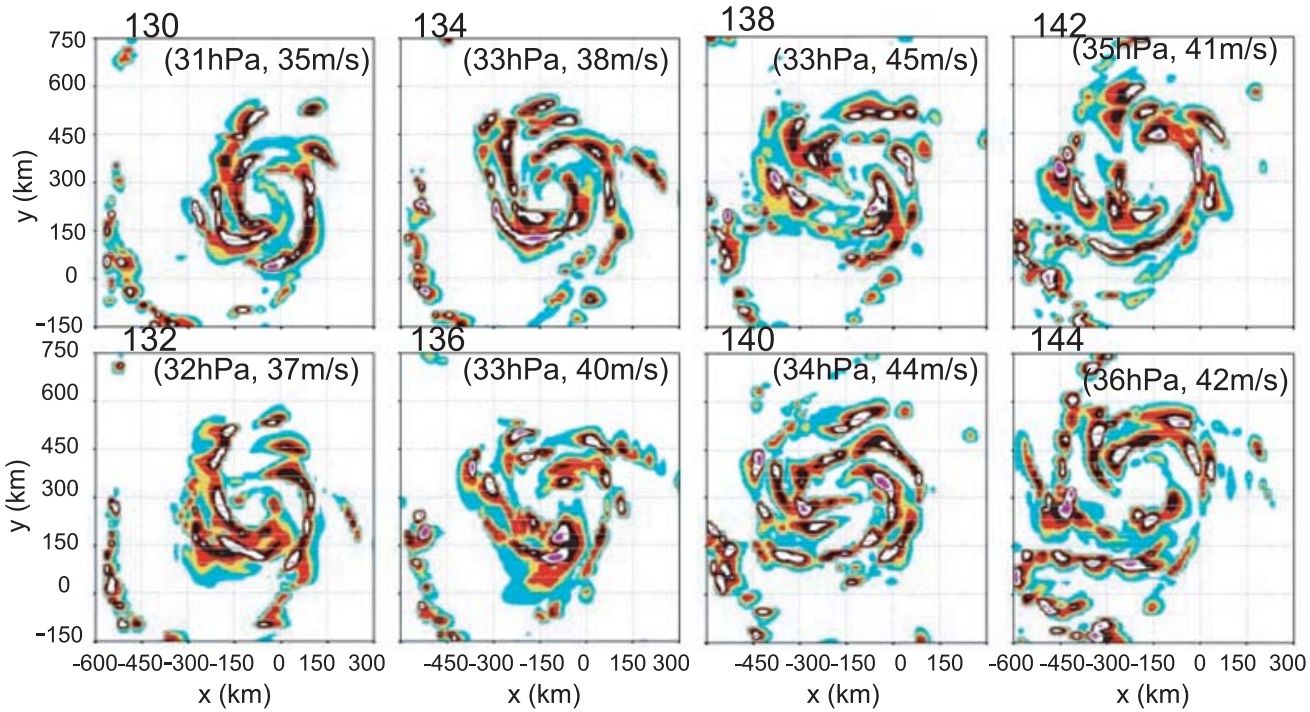

Fig. 5. Rainwater mixing ratio near the surface at an interval of $2 \mathrm{~h}$ from $130 \mathrm{~h}$ to $144 \mathrm{~h}$ in case 3 . 
subgrid-scale effect is excluded after $96 \mathrm{~h}$ in case 2 . It is seen that the eyewall, which is, to a fair degree, simulated in case 2, is largely distorted and it is not well defined in case 3 . The anomaly of the central surface pressure is $-31 \mathrm{hPa}$ and $-36 \mathrm{hPa}$ at 130 and $144 \mathrm{~h}$, respectively. The maximum wind speed increases from 35 to $45 \mathrm{~m} \mathrm{~s}^{-1}$ in a period of 130 $138 \mathrm{~h}$. Compared with the result for case 2 , it can be confirmed that the exclusion of the subgrid-scale convection suppresses the development of the TC. This is consistent with the result obtained from the comparison of Fig. 1 with Fig. 2. Some researchers may argue that the stronger TC in case 2 is not necessarily reasonable. However, the degree of latent instability (buoyancy of rising air) given at the initial time and the surface heat flux associated with the intensified surface wind of the vortex may suggest that the maximum TC intensity $(-56 \mathrm{hPa}$ and $57 \mathrm{~m} \mathrm{~s}^{-1}$ ) in case 2 should be reasonable. Numerical experiments with a CCRM (with a grid size of $1 \mathrm{~km}$ or less) are desirable to confirm this.

In connection with the difference in the TC intensities, the movement (speed and direction) of the TC center is also different between case 1 (case 3) and case 2. The movement speed is fairly smaller in case 1 because of the smaller beta-drift associated with the weaker TC.

\subsection{Structure of the TC in the MCRM}

In the following, we examine the cloud field at the mature stage in case 2 . As an example, the total mixing ratio of cloud water and cloud ice at five levels at $138 \mathrm{~h}$ is shown in Fig. 6a (left panels). For comparison, rainwater mixing ratio at a height of $150 \mathrm{~m}$ is reproduced in Fig. 6f (lowest right panel). It is seen that an eyewall and a spiral pattern of clouds are simulated. A large portion of the clouds consists of convective clouds. In the upper troposphere, stratiform clouds are also seen, although stratiform clouds may tend to be confined to upper levels (above $8 \mathrm{~km}$ ) than those observed. The mixing ratio is also smaller because the coarse grid is used, and averaged values for the grid are predicted. The subgrid-scale cloud water field is shown in Fig. 6b. A fairly large portion of subgridscale cloud water extends to upper levels such as $10.0 \mathrm{~km}$ or $12.5 \mathrm{~km}$ because of the positive buoyancy of rising air as mentioned below.

One of important features to be described here is the ratio of the mixing ratio of subgrid-scale cloud water to the total mixing ratio of cloud water and cloud ice. This ratio is shown in Fig. 6c. The blue color indicates areas where subgrid-scale cloud water does not exist, although grid-resolved clouds exist. The red color indicates that the ratio is larger than 0.8 , which means that the cloud primarily consists of the subgrid-scale. In areas where this ratio is 1.0, the two patterns in Fig. 6a and Fig. $6 \mathrm{~b}$ are the same. This area is most pronounced in the middle troposphere, and in the eyewall and spiral rainbands. The latter feature is consistent with the result that the difference between TCs in case 1 and case 2 is large in areas of the eyewall and spiral rainbands. In the upper troposphere and near the cloud base, grid-resolved condensation contributes much to the cloud field, as expected.

The buoyancy fields at three levels are shown in Fig. 6d. A measure of buoyancy is defined (with a unit of temperature) by (24) in subsection 2.4. In all figures in the previous papers, the author has used a measure of buoyancy that is defined in terms of the equivalent potential temperature $\theta_{e}$ at some level in the boundary layer and the saturated value of $\theta_{e}$ at each level. In this paper, a measure of buoyancy in which the entrainment effect is taken into account is shown in the figures. Although the difference between the two definitions is not large, the present definition should be more desirable because it is a measure of actual buoyancy. Figure $6 \mathrm{~d}$ indicates that the buoyancy at $2.7 \mathrm{~km}$ is positive in almost entire area shown in this figure, and the buoyancy at $12.5 \mathrm{~km}$ is still positive in the inner area of the TC (areas of the eyewall and inner spiral rainbands).

The equivalent potential temperature $\theta_{e}$ at a height of $800 \mathrm{~m}$, which is taken to be the origin of the rising air, is shown in Fig. 6e. The pattern is similar to what is expected. The area of higher $\theta_{e}$ in the southwestern corner of the area is not necessarily present in many cases using different initial conditions. In addition, since the moist area is taken to be wide even to the north of the initial vortex center, the intrusion of air with lower values of $\theta_{e}$, which is usually typical of observed TCs, is not pronounced in case 2 . The $\theta_{e}$ field at a height of $150 \mathrm{~m}$ (not shown) is significantly different from that at $800 \mathrm{~m}$, as expected. The effects of downdraft and cooling due to evaporation of rainwater are pronounced at this level, as was shown in the 1980 s by numerical and observational studies (e.g., Yamasaki 1983; Barnes et al. 1983).

As for the low-level rainwater field, it is most correlated with the cloud field in the middle troposphere (at $6.2 \mathrm{~km}$ in Fig. 6a), as expected. There 
case $2138 \mathrm{~h}$

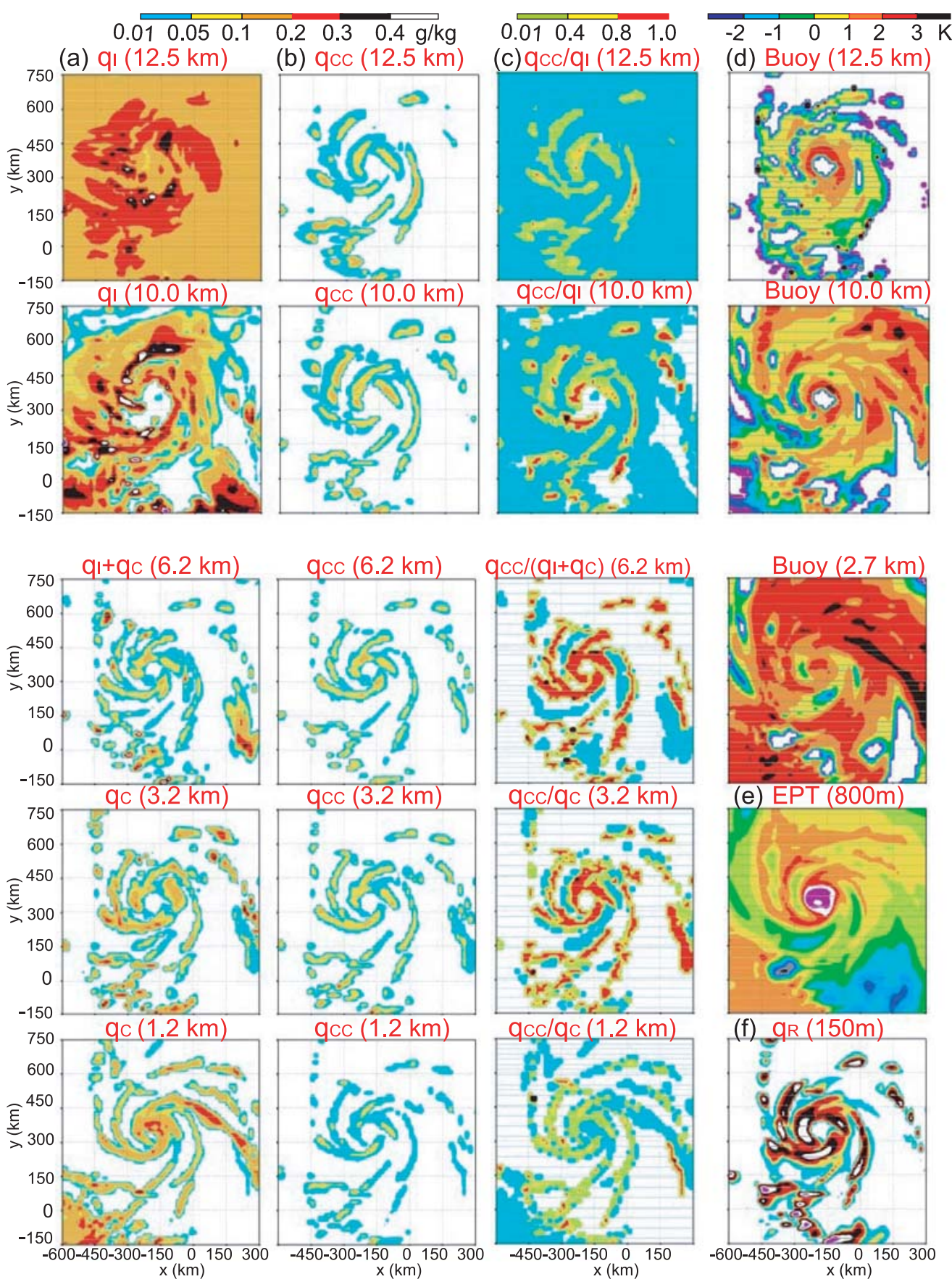

Fig. 6. (a) Mixing ratio of the sum of cloud ice and cloud water $\left(q_{I}+q_{C}\right)$, (b) subgrid-scale cloud water $\sigma q_{C}^{C}$, (c) ratio of subgrid-scale cloud water to the sum, $\sigma q_{C}^{C} /\left(q_{I}+q_{C}\right)$ at five levels, (d) buoyancy of rising air at three levels, (e) equivalent potential temperature $\theta_{e}$ at $800 \mathrm{~m}$, and (f) rainwater $q_{R}$ near the surface. The blue area in (c) indicates that $q_{C}^{C}$ is 0 but $\left(q_{I}+q_{C}\right)$ is not 0 . The purple color in (e) indicates that $\theta_{e}$ is higher than $350 \mathrm{~K}$. Different colors are used at an interval of $2 \mathrm{~K}$. 
(a) W-E cross section at $\mathrm{y}=340 \mathrm{~km}$ case $2138 \mathrm{~h}$

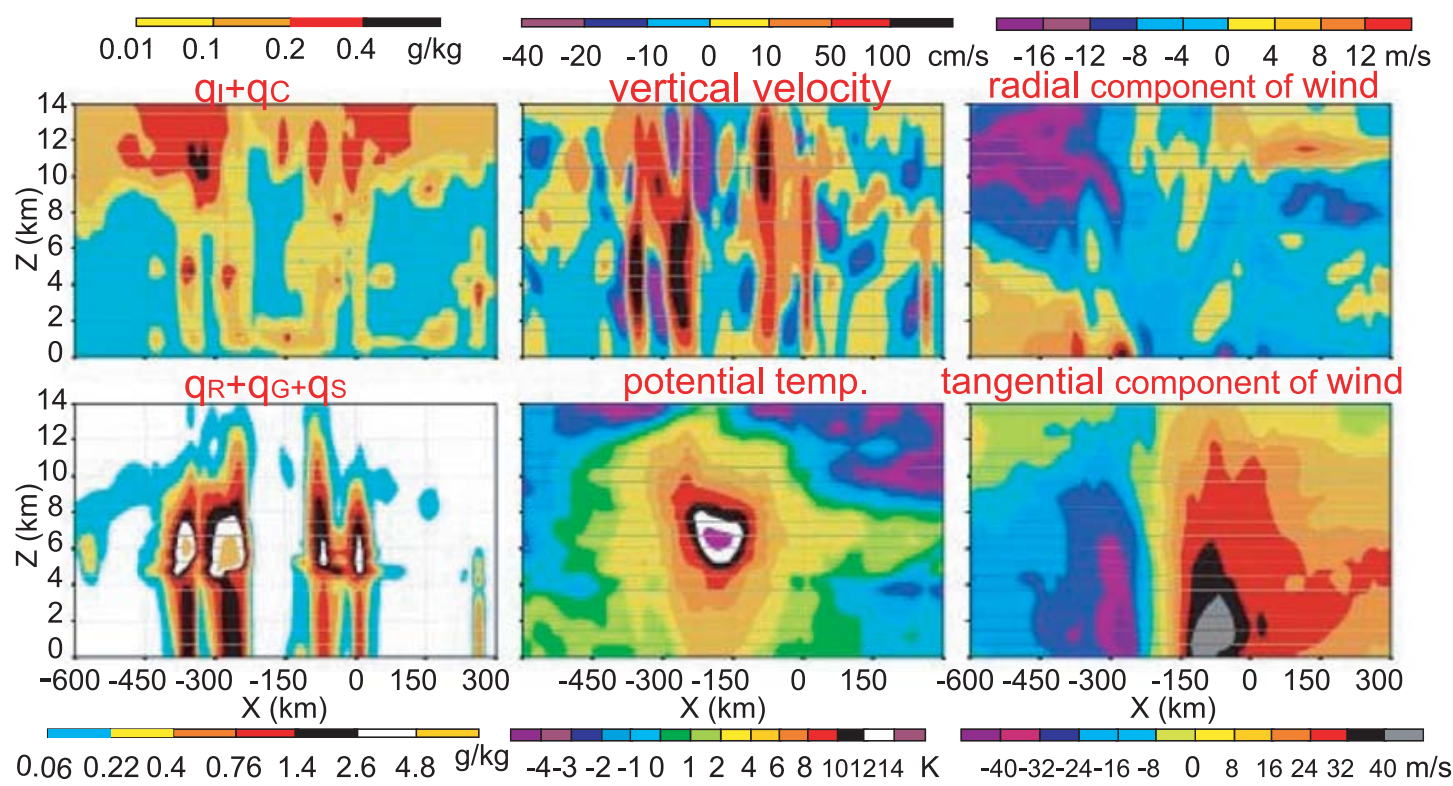

(b) S-N cross section at $x=-200 \mathrm{~km}$
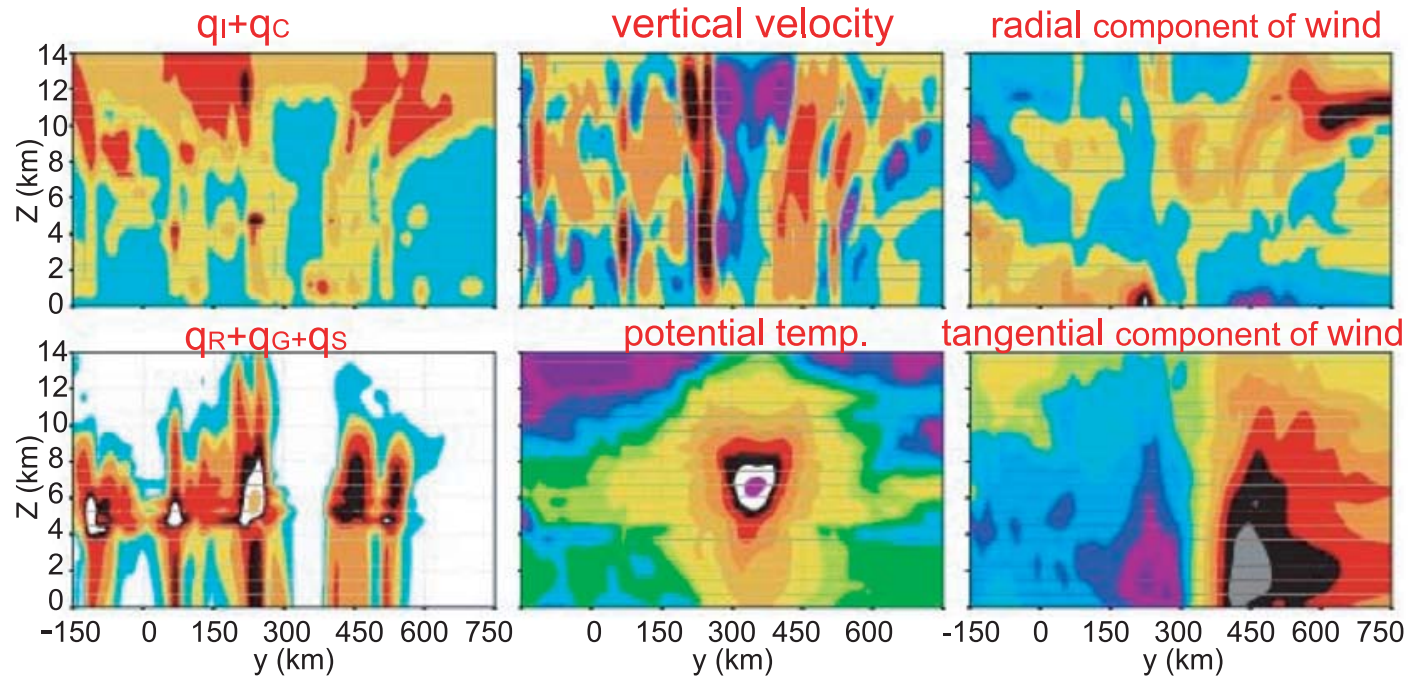

Fig. 7. Vertical structure of a tropical cyclone at $138 \mathrm{~h}$ in case 2. (a) West-east and (b) south-north cross sections crossing the center of the TC. The total mixing ratio of cloud ice and cloud water $\left(q_{I}+q_{C}\right)$ and that of rainwater, graupel, and snow $\left(q_{R}+q_{G}+q_{S}\right)$, vertical velocity, potential temperature anomaly, and radial and tangential components of the wind vector are shown.

exist shallow clouds which do not produce significant rainwater and whose top is below or around $3 \mathrm{~km}$, as seen from the comparison with the lower left panels.

Two examples of the vertical structure in case 2 are shown in Fig. 7. The west-east and south- north sections crossing the center of the TC at $138 \mathrm{~h}$ (corresponding to Fig. 6) are shown for the total mixing ratio of cloud water and cloud ice, that of rainwater, graupel and snow (snow aggregate), vertical velocity, potential temperature anomaly, and radial and tangential components of the 
wind speed. Many features that have been observed and simulated by numerical experiments in the past can be seen: strong upward motion in the eyewall cloud, outward tilt of the eyewall, the upper stratiform cloud, upward motion in most portions of the stratiform cloud, snow and graupel inside and below the stratiform cloud, a warm core centered at the eye, a slightly cool layer corresponding to the melting layer (about 4.5-km height), low-level inflow concentrated near the surface (frictional flow), upper-level outflow centered around $12-\mathrm{km}$ height, mid-to-upper level inflow around $8-10-\mathrm{km}$ height just below the upper-level outflow, cyclonic rotation that extends to the upper troposphere, etc. A pronounced asymmetry is seen between the southern and northern portions of the cross section, as very often observed in TCs. The center of the warm core is located at about 6-km height, which is somewhat lower compared with that of intense TCs. The anticyclonic rotation, which is observed at some distance from the TC center in the upper troposphere, can be clearly seen to the south of the TC center. Mesoscale subsidence and rainfall just outside the eyewall and cooling near the surface (associated with rainbands) are not pronounced in the figure although these features can be seen at other periods. The cold pool associated with rainbands plays an important role in most cases, as mentioned above. In contrast to the TC in case 2, the vertical structures in case 1 and case 3 do not represent the eye-eyewall feature, as shown in Fig. 3a and Fig. 5.

\subsection{Additional discussions}

Numerical experiments have also been performed with a weaker initial vortex having the maximum wind speed of $5 \mathrm{~m} \mathrm{~s}^{-1}$. Figures $8 \mathrm{a}, \mathrm{b}$ show the surface rainfall intensity at an interval $6 \mathrm{~h}$ from $102 \mathrm{~h}$ to $132 \mathrm{~h}$ in case $1 \mathrm{~V}$ (without the subgrid-scale effect) and in case $2 \mathrm{~V}$ (with the effect), respectively. As in case 1, an eyewall and spiral rainbands are not simulated in case $1 \mathrm{~V}$. More precisely, the simulation of the eyewall and rainbands is worse than in case 1 because the initial vortex in case $1 \mathrm{~V}$ is weaker than in case 1 . This is just what was expected. On the other hand, these are simulated to a fair degree in case $2 \mathrm{~V}$. Because of the weaker initial vortex, the development of the TC in terms of the central surface pressure and the maximum wind speed delays about $12 \mathrm{~h}$ in case $2 \mathrm{~V}$, compared with that in case 2 . The anomaly of the central surface pressure changes from $-13 \mathrm{hPa}$ to $-18 \mathrm{hPa}$ in case
$1 \mathrm{~V}$ and from $-21 \mathrm{hPa}$ to $-41 \mathrm{hPa}$ in case $2 \mathrm{~V}$ in the period from 102 to $132 \mathrm{~h}$. The TC in case $2 \mathrm{~V}$ continues to develop after $132 \mathrm{~h}$, and the eyewall and spiral rainbands behave (more) realistically (not shown).

Further numerical experiments have been performed without any vortex, but with four buoyancy perturbations on a circle with a radius of $50 \mathrm{~km}$ at the initial time. The maximum anomaly of temperature is taken to be $1 \mathrm{~K}$, and the radius of each anomaly is $40 \mathrm{~km}$. The radial profile is taken to be the square of cosine. The relative humidity field is the same as that in the other cases, and therefore, buoyancy comes from not only temperature but also water vapor. Figures $9 \mathrm{a}, \mathrm{b}$ show the surface rainfall intensity at an interval of $6 \mathrm{~h}$ in a period from $126 \mathrm{~h}$ to $168 \mathrm{~h}$ (except for $150 \mathrm{~h}$ ) in case 1T (without the subgrid-scale effect) and in case 2T (with the effect), respectively. As in the case of the imposed initial vortex, an outward propagating, ring-shaped rainband is produced by the initial buoyancy perturbations. A portion of the ringshaped rainband can be seen at $126 \mathrm{~h}$ and $132 \mathrm{~h}$ in both cases. As in case 1V, the eyewall and spiral rainbands are not simulated in case $1 \mathrm{~T}$. On the other hand, these are simulated in case $2 \mathrm{~T}$, although their behaviors are different from those in case 2 and case $2 \mathrm{~V}$.

As mentioned above, the process of the TC formation is controlled somewhat artificially in all the numerical experiments presented in this paper. However, the author strongly feels that these experiments are very useful to the primary objectives of this study, on the basis of his studies in these 40 years. Numerical experiments have also been performed to simulate and understand the TCformation process under realistic conditions. Together with numerical experiments with the hydrostatic MCRM (Yamasaki 1988, 1989a, 2006a, b), these experiments suggest that the formulation concerning the subgrid-scale effects of cumulus convection described in this paper can also be applied to the process of TC formation. Results will be reported in a separate paper.

\section{Concluding remarks}

This paper describes a nonhydrostatic version of the mesoscale-convection-resolving model (MCRM) of Yamasaki $(1986,2001)$ and some evaluation of the model performance on the basis of numerical experiments under idealized (simplified) initial conditions. The background for the develop- 

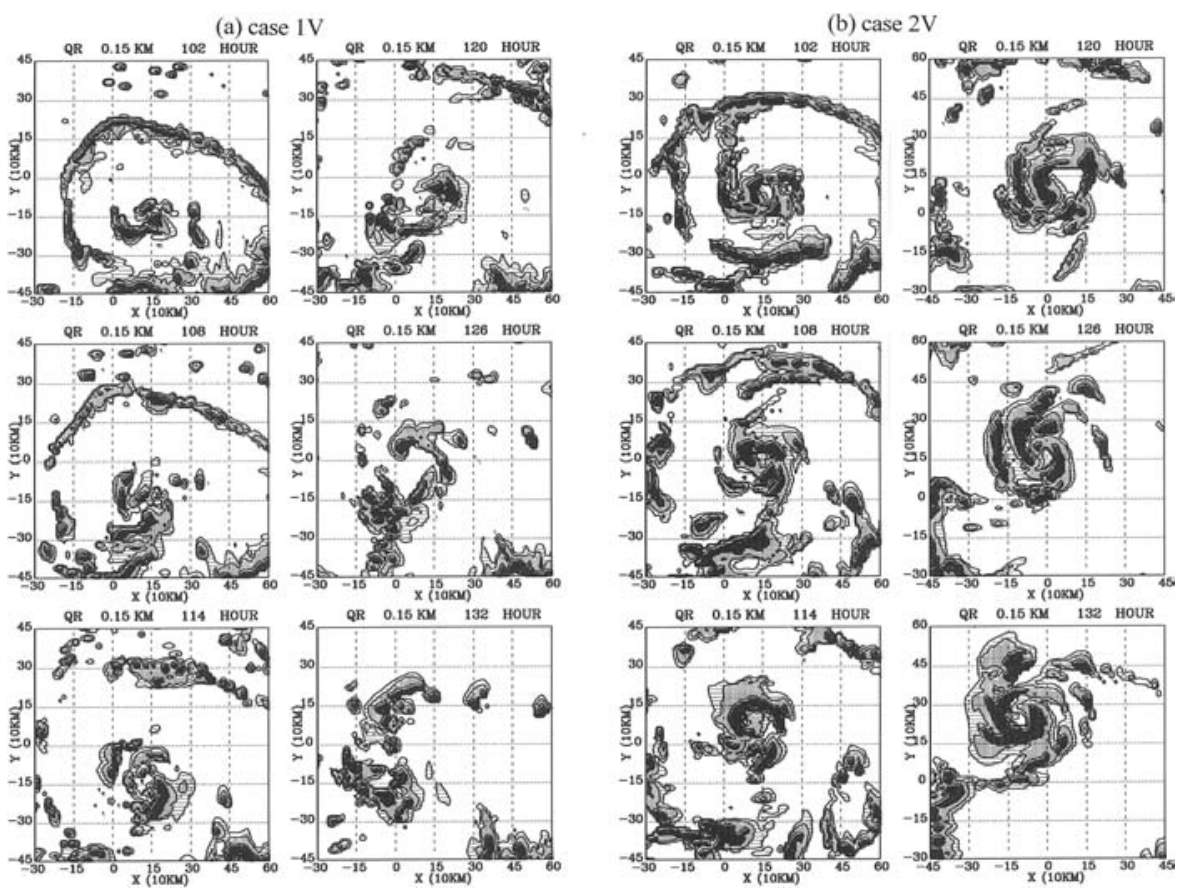

Fig. 8. Rainwater mixing ratio at a height of $150 \mathrm{~m}$, or the surface rainfall intensity at an interval of $6 \mathrm{~h}$ from $102 \mathrm{~h}$ to $132 \mathrm{~h}$ in (a) case $1 \mathrm{~V}$ and (b) case $2 \mathrm{~V}$. In these experiments, the maximum tangential velocity of the initial vortex is taken to be $5 \mathrm{~m} \mathrm{~s}^{-1}$. The different shades indicate rainfall intensities stronger than 16, 4 , and $1 \mathrm{~mm} \mathrm{~h}^{-1}$. The areas shown on the right and left in (b) are different.
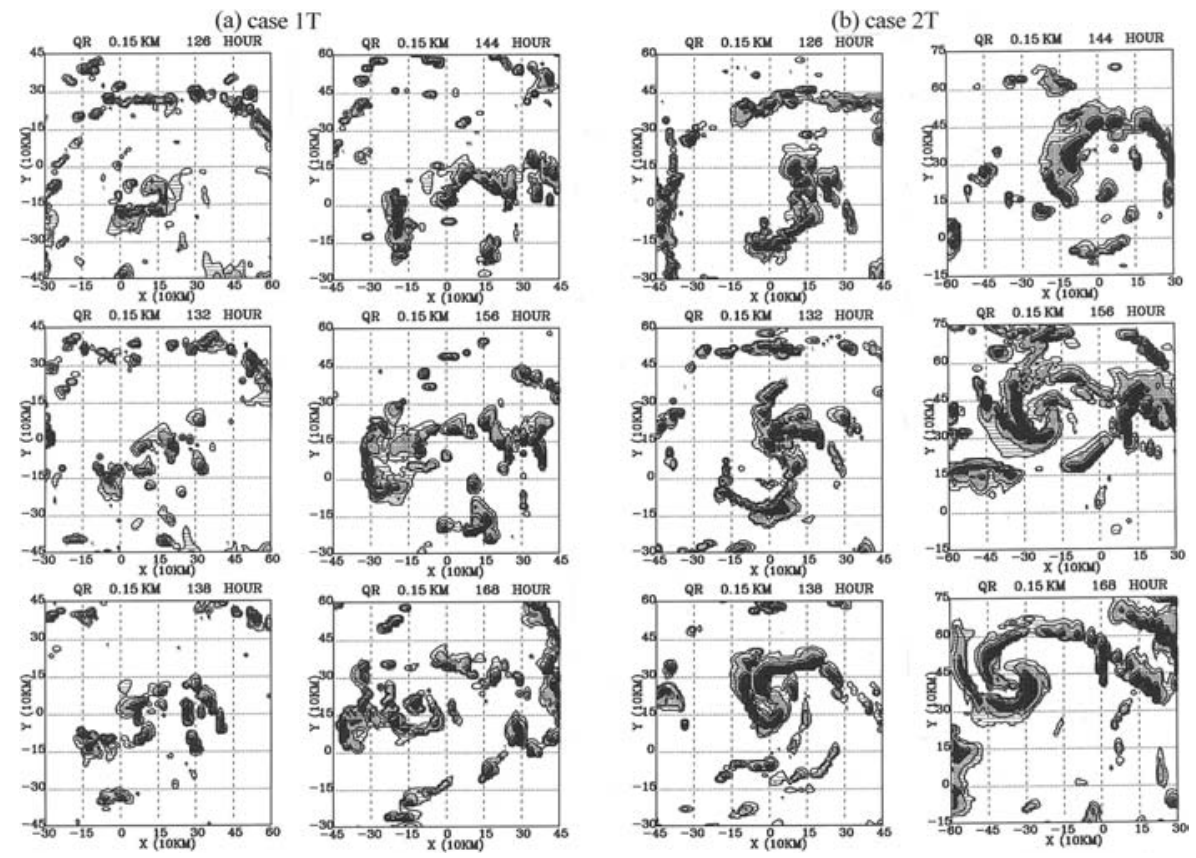

Fig. 9. Same as Fig. 8 except for (a) case $1 \mathrm{~T}$ and (b) case $2 \mathrm{~T}$ in which a buoyancy perturbation (instead of a vortex) is given at the initial time. The areas shown are different, depending on the location of the TC center. 
ment of the MCRM and the specific meaning of mesoscale convection (MC) are described in some detail in Section 1.

A cumulus-convection-resolving model with a horizontal grid size of $1 \mathrm{~km}$ or less has a wider application in recent years because of the advancement of computers, and there is no doubt that its applicability will greatly increase in coming years. On the other hand, a model with a coarser grid such as MCRMs will be still very useful for efficient research and numerical weather prediction. In order that such a coarse-grid model may be more useful, the formulation of the subgrid-scale effects of cumulus convection should be appropriate. Although the formulation described in this paper is not satisfactory yet, the author strongly feels that it can be considered as an important step toward a satisfactory model. As mentioned in Yamasaki (2007a), the author has felt that there would be no better way other than the development of the MCRM in which only cumulus convection is treated as the subgrid-scale and MC is resolved by the grid of a numerical model.

Some researchers may argue that they hesitate to use the MCRM because the model includes several uncertain parameters (and physical processes)*. The author has hoped that uncertain physical processes should be clarified through basic research, and thereby, the model be improved. The author has also expected improvements in the MCRM through interactions with other researchers in these 20 years. However, there have been no interactions. It appears that many of researchers have not been interested in the MCRM. Therefore, most of the papers referred in this paper are the author's. (The author knows that a number of studies have been made to simulate the gross features of TCs and understand their mechanisms in these 50 years (e.g., Anthes 1982; Yamasaki 1982). Many of discussions concerning the mechanisms of TCs (related to many past studies) are not included in this paper to avoid a lengthy paper. This problem will be discussed in a separate paper when it is necessary.)

As a preliminary step of this study, numerical

* One of advantages of the MCRM is that it includes formulations of many individual physical processes (in contrast to other models in the past). As a result, the model includes a larger number of uncertain parameters. This can be considered as rather desirable because the model can be improved more easily through better understanding of individual physical processes. experiments were performed with a horizontal grid size of $2 \mathrm{~km}$, nearly corresponding to that of a cumulus-convection-resolving model (CCRM). Results from these experiments are not included in this paper partly because a much smaller domain was used. As mentioned in section 1, a comparison of the results from the MCRM with those from a CCRM is one of important problems to evaluate the performance of the MCRM. In this case, it is also important to know the degree of validity of the CCRM. Comparison with the results from a CCRM having a large domain, which is comparable to that used in this study, remains to be made in the future. It is strongly hoped that results from CCRMs with a horizontal grid size of $1 \mathrm{~km}$ or less will be reported by many researchers, which will also be very useful to improve the MCRM.

It should be emphasized that the hydrostatic MCRM will be still very useful even after the development of the nonhydrostatic MCRM described in this paper has been achieved. The MCRM was developed on the basis of the preposition that the model can be used most efficiently for horizontal grid sizes of $20-5 \mathrm{~km}$. Except for problems concerning the fine structure (such as convective-scale) of TCs, the hydrostatic MCRM has important advantages over the nonhydrostatic; that is, basic research and numerical weather prediction can be made with a much smaller computer time (CPU time) because sound waves and external gravity waves are avoided. In addition, when one deals with problems in which the orography is important, the use of sigma coordinates is easier in the hydrostatic model. The author presented results from numerical experiments with the hydrostatic MCRM (with a horizontal grid size of $5 \mathrm{~km}$ ) to discuss the diurnal cycle of organized convection and rainfall over a large island in the equatorial area (presented at the spring meeting of the Meteorological Society of Japan in 2007). The phase of the diurnal cycle of rainfall was much improved compared with that from other models. Recently, the author has used the hydrostatic MCRM (with a grid size of about $20 \mathrm{~km}$ ), aiming at a better simulation and understanding of the Madden-Julian oscillation (including wave CISK and the hierarchical structure consisting of super cloud cluster, cloud cluster and $\mathrm{MC})$. The nonhydrostatic version requires much more computer time for this problem.

Finally, it is added that the author has used two types of models, CCRM and hydrostatic MCRM, and will use three types of models, including the 
nonhydrostatic MCRM (in addition to the two), for studies of various problems, depending on the objectives of research. The MCRM was developed in the 1980s with an intention of improving numerical models for studies of not only TCs but also other phenomena in which moist convection plays an important role. In addition, studies with the CCRM applied to various problems (e.g., Yamasaki 2004, 2007a, 2009) as well as observations will still be very important to understand the observed phenomena and to get a basis for improving the two MCRMs.

\section{Acknowledgments}

The numerical experiments have been performed by using the NEC SX-8R computer of the Japan Agency for Marine-Earth Science and Technology.

\section{References}

Anthes, R. A., 1972: Development of asymmetries in a three-dimensional numerical model of the tropical cyclone. Mon. Wea. Rev., 100, 461-476.

Anthes, R. A., 1982: Tropical cyclones-their evolution, structure and effects. Meteor. Monographs, 19, No. 41, Amer. Meteor. Soc., 208pp.

Anthes, R. A., S. L. Rosenthal, and J. W. Trout, 1971: Preliminary results from an asymmetric model of the tropical cyclone. Mon. Wea. Rev., 99, 744-758.

Arakawa, A., and W. H. Schubert, 1974: Interaction of a cumulus cloud ensemble with the large-scale environment. Part I. J. Atmos. Sci., 31, 674-701.

Barnes, G. M., E. J. Zipser, D. Jorgensen, and F. Marks, Jr., 1983: Mesoscale and convective structure of a hurricane rainband. J. Atmos. Sci., 40, 2125-2137.

Berry, E. X., 1968: Modification of the warm rain process. Proc. First National Conf. Weather Modification, Amer. Meteor. Soc., Boston, Mass., 81-85.

Charney, J. G., and A. Eliassen, 1964: On the growth of the hurricane depression. J. Atmos. Sci., 21, $68-75$.

Kessler, E., 1969: On the distribution and continuity of water substance in atmospheric circulaion. Meteor. Monographs, 10, No. 32, Amer. Meteor. Soc., 84pp.

Kuo, H. L., 1965: On formation and intensification of tropical cyclones through latent heat release by cumulus convection. J. Atmos. Sci., 22, 40-63.

Kurihara, Y., and R. E. Tuleya, 1974: Structure of a tropical cyclone developed in a three-dimensional numerical simulation model. J. Atmos. Sci., 31, 893-919.

Leary, C. A., and R. A. Houze, Jr., 1979: The structure and evolution of convection in a tropical cloud cluster. J. Atmos. Sci., 36, 437-457.
Nasuno, T., and M. Yamasaki, 2001: A representation of cumulus-scale effects in a mesoscale-convectionresolving model for tropical cyclones. J. Meteor. Soc. Japan, 79, 1035-1057.

Ooyama, K., 1964: A dynamical model for the study of tropical cyclone development. Geophys. Internacional, 4, 187-198.

Ooyama, K., 1969: Numerical simulation of the life cycle of tropical cyclones. J. Atmos. Sci., 26, 3-40.

Ooyama, K., 1971: A theory for parameterization of cumulus convection. J. Meteor. Soc. Japan, 49 (special issue), 744-756.

Ooyama, K. V., 1982: Conceptual evolution of the theory and modeling of the tropical cyclone. J. Meteor. Soc. Japan, 60, 369-379.

Ooyama, K. V., 1990: A thermodynamic foundation for modeling the moist atmosphere. J. Atmos. Sci., 47, 2580-2593.

Ooyama, K. V., 2001: A dynamic and thermodynamic foundation for modeling the moist atmosphere with parameterized microphysics. J. Atmos. Sci., 58, 2073-2102.

Satoh, M., 2003: Conservative scheme for a compressible nonhydrostatic model with moist processes. Mon Wea. Rev., 131, 1033-1050.

Syono, S., and M. Yamasaki, 1966: Stability of symmetrical motions driven by latent heat released by cumulus convection under the existence of surface friction. J. Meteor. Soc. Japan, 44, 353-375.

Tatehira, R., 1961: A mesosynoptic and radar analysis of typhoon rain band (case study of Typhoon "Helen", 1958). Proc. Second Tech. Conf. Hurricanes, Amer. Meteor. Soc., 115-126.

Tatehira, R., 1962: Radar and meso-scale analysis of rainband in Typhoon Georgia in 1959-comparison with the case of Typhoon Helen in 1958 - Kenkyu Jiho, 14, 621-630 (in Japanese).

Yamasaki, M., 1968a: Numerical simulation of tropical cyclone development with the use of primitive equations. J. Meteor. Soc. Japan, 46, 178-201.

Yamasaki, M., 1968b: A tropical cyclone model with parameterized vertical partition of released latent heat. J. Meteor. Soc. Japan, 46, 202-214.

Yamasaki, M., 1968c: Detailed analysis of a tropical cyclone simulated with a 13-layer model. Papers Meteor. Geophys., 19, 559-585.

Yamasaki, M., 1969: Large-scale disturbances in the conditionally unstable atmosphere in low latitudes. Papers Meteor. Geophys., 20, 289-336.

Yamasaki, M., 1971: A further study of wave disturbances in the conditionally unstable model tropics. J. Meteor. Soc. Japan, 49, 391-415.

Yamasaki, M., 1975: A numerical experiment of the interaction between cumulus convection and largerscale motion. Papers Meteor. Geophys., 26, 63-91.

Yamasaki, M., 1977a: A preliminary experiment of the 
tropical cyclone without parameterizing the effects of cumulus convection. J. Meteor. Soc. Japan, 55, 11-31.

Yamasaki, M., 1977b: The role of surface friction in tropical cyclones. J. Meteor. Soc. Japan, 55, 559572.

Yamasaki, M., 1979: A further study of a CISK mode unaffected by surface friction. J. Meteor. Soc. Japan, 57, 112-132.

Yamasaki, M., 1982: Typhoon. Tokyodo Pub., 206pp (in Japanese).

Yamasaki, M., 1983: A further study of the tropical cyclone without parameterizing the effects of cumulus convection. Papers Meteor. Geophys., 34, $221-260$.

Yamasaki, M., 1984: Dynamics of convective clouds and 'CISK' in vertical shear flow-with its application to easterly waves and squall-line systems. J. Meteor. Soc. Japan, 62, 833-863.

Yamasaki, M., 1986: A three-dimensional tropical cyclone model with parameterized cumulus convection. Papers Meteor. Geophys., 37, 205-234.

Yamasaki, M., 1987: Parameterization of cumulus convection in a tropical cyclone model. Collection of papers presented at the WMO/IUGG NWP Symposium, Tokyo, 1986, J. Meteor. Soc. Japan, (special volume), 65, 665-678.

Yamasaki, M., 1988: Towards an understanding of the interaction between convection and the larger-scale in the tropics. Aust. Meteor. Mag., 36, 171-182.

Yamasaki, M., 1989a: Numerical experiment of tropical cyclone formation in the intertropical convergence zone. J. Meteor. Soc. Japan, 67, 529-540.

Yamasaki, M., 1989b: Advances in basic understanding of tropical cyclone formation, The second WMO international workshop on tropical cyclones, Manila, WMO Tropical Meteorology Research Programme Report Series, WMO/TD-No. 319, Vol. $1,125-142$.

Yamasaki, M., 1992: A study of tropical cyclone motion with a nested-grid model including rainwater pre- diction. Papers Meteor. Geophys., 43, 61-77.

Yamasaki, M., 1996: A study on CISK and cloud systems. Kisho-Kenkyu Note, 186, 75-96 (in Japanese).

Yamasaki, M., 2001: A tropical cyclone model resolving mesoscale organized convection with prognostic treatment of subgrid-scale cloud water. J. Meteor. Soc. Japan, 79, 637-655.

Yamasaki, M., 2002: A study of cloud clusters associated with a Baiu front by use of a mesoscaleconvection-resolving model. J. Meteor. Soc. Japan, 80, 595-619.

Yamasaki, M., 2004: A study of the 22 February 1993 TOGA COARE squall line: A case used for the GCSS model intercomparison. J. Meteor. Soc. Japan, 82, 1331-1363.

Yamasaki, M., 2005a: A numerical study of cloud clusters and a meso- $\alpha$-scale low associated with a Meiyu front. J. Meteor. Soc. Japan, 83, 305-329.

Yamasaki, M., 2005b: A study of the mesoscale structure of Typhoon Flo (T9019): A case of COMPARE model intercomparison. J. Meteor. Soc. Japan, 83, 1057-1084.

Yamasaki, M., 2006a: A study of tropical cyclone formation with a $20-\mathrm{km}$ grid model. SOLA, 2, 9-12.

Yamasaki, M., 2006b: A numerical study of tropical cyclone formation from two mesoscale convective systems in a large-scale convective system. SOLA, 2, 57-60.

Yamasaki, M., 2007a: A view on tropical cyclones as CISK. J. Meteor. Soc. Japan, 85, 145-164.

Yamasaki, M., 2007b: A numerical study of rainfall associated with a Baiu front: A case of NiigataFukushima heavy rainfall in 2004. SOLA, 3, 5760.

Yamasaki, M., 2008: A study of Fukui heavy rainfall in 2004. J. Meteor. Soc. Japan, 86, 369-376.

Yamasaki, M., 2009: A study of the mesoscale convective system under vertical shear flow in the latently unstable atmosphere with north-south asymmetry. J. Meteor. Soc. Japan, 87, 245-262. 